\title{
Mode de conduite en élevage et tendreté de la viande bovine
}

\author{
M.-P. OURY', B. PICARD ${ }^{2}$, L. ISTASSE 3 , D. MICOL ${ }^{2}$, R. DUMONT' \\ ${ }^{1}$ ENESAD, Equipe Système d'Elevage et Qualité des Animaux et des Viandes, F-21079 Dijon, France \\ 2 INRA, UR1213 Herbivores, F-63122 Saint-Genès Champanelle, France \\ ${ }^{3}$ Faculté de Médecine Vétérinaire, Service de Nutrition des Animaux Domestiques, 20 Bd de Colonster, \\ B-4000 Liège, Belgique \\ Courriel : mp.oury@enesad.fr
}

\begin{abstract}
La tendreté apparaît comme un critère déterminant pour la viande rouge qui est consommée surtout sous forme grillée ou rôtie. Sa variabilité élevée, mal perçue par le consommateur, peut être attribuée à la fois aux facteurs technologiques et aux facteurs d'élevage. Ces derniers peuvent être ainsi analysés dans des conditions d'abattage et de conservation des viandes connues et maîtrisées.
\end{abstract}

En France, la viande de gros bovins est un produit très courant de la consommation alimentaire des ménages représentant $25 \%$ de la consommation totale des produits carnés en 2005. Mais sa part a tendance à diminuer régulièrement depuis les années 1980 , de $33 \mathrm{~kg}$ équivalent-carcasse/habitant/an en 1980 à $23 \mathrm{~kg}$ équivalent-carcasse/habitant/an environ en 2005 (Office de l'élevage 2006). Elle a, en effet tendance à être délaissée par des consommateurs qui cherchent à limiter les apports de matières grasses d'origine animale dans leur alimentation (Combris 2003). De plus, la modification actuelle des modes de vie conduit de plus en plus les consommateurs à orienter leurs achats vers des produits dont la préparation et la cuisson ne demandent qu'un minimum de temps. Ainsi, l'évolution récente de la consommation est marquée par le clivage entre d'une part les viandes hachées en progression et d'autre part, les morceaux à griller, à rôtir, à bouillir ou à braiser en recul (Office de l'élevage 2006). Cependant, les achats des ménages sont plutôt tournés vers les viandes à griller ou à rôtir. Elles représentent près de $60 \%$ des achats de viandes de boucherie en 2005, contre $20 \%$ pour les viandes à cuisson lente ou encore les viandes hachées.

La préférence des consommateurs pour les viandes à cuisson rapide laisse supposer qu'au sein des descripteurs sensoriels, l'exigence porte prioritairement sur la tendreté de la viande. Or, certaines enquêtes ont mis en évidence que le défaut le plus généralement évoqué concernant la viande rouge est l'irrégularité de la qualité sensorielle et en particulier de la tendreté (Tarrant 1998, Bindon et Jones 2001). Ce phénomène est préoccupant dans la mesure où cette variabilité est susceptible de conduire à une insatisfaction du client qui pourrait exacerber sa tendance à se détourner de la viande bovine, alors même que certains consommateurs semblent prêts à payer plus cher un morceau de tendreté garantie (Boleman et al 1997).

Consciente de cette difficulté, la filière cherche à mettre à la disposition de sa clientèle des viandes de qualité sensorielle satisfaisante et régulière. Parmi les facteurs susceptibles d'influer sur la qualité sensorielle des viandes, les facteurs technologiques, à savoir les conditions d'abattage, de réfrigération et de maturation, jouent un rôle prépondérant (Ouali 1991). Cependant, lorsque ces facteurs sont maîtrisés, il demeure une variabilité de la qualité sensorielle des viandes et notamment de la tendreté. On peut donc s'interroger sur l'influence des conditions d'élevage d'autant que chez le bovin, les schémas de production sont très diversifiés à la différence du porc ou de la volaille. Ainsi, les différences régionales qu'elles concernent, les conditions climatiques, les ressources alimentaires, les races exploitées, les types d'animaux utilisés ou encore la nature de la demande des marchés et des débouchés, conduisent à produire des carcasses de bovin présentant des caractéristiques qualitatives très variées.
L'objet de cette revue bibliographique est d'analyser l'influence des principaux facteurs d'élevage sur la tendreté de la viande bovine. Parmi ces facteurs de variation, la race et le sexe ont été assez étudiés. Les travaux qui ont comparé la tendreté de la viande d'animaux de races différentes (Maltin et al 2003) permettent de conclure que les différences de qualité des viandes entre races ne sont généralement pas significatives, compte-tenu de la forte variabilité individuelle observée au sein même d'une race (Renand 1988). C'est cette variabilité intra-race qu'il convient de réduire pour maîtriser la qualité sensorielle. Concernant l'effet du sexe, on admet généralement que les mâles entiers produisent des viandes de tendreté inférieure à celles des mâles castrés, elle-même inférieure à celles de génisses. Ce résultat a pu être expliqué par des teneurs en collagène total significativement inférieures pour les muscles de femelle comparativement aux mâles (Hocquette et al 2005). Audelà de ces deux facteurs principaux de variation, il reste à vérifier si les autres paramètres d'élevage peuvent également influer sur la qualité de la viande et notamment la tendreté. Pour une catégorie d'animal (jeune bovin, bœuf, génisse, vache) et une race donnée, ces paramètres concernent :

- l'animal lui-même (le tri des animaux à travers l'étude du type morphologique, l'âge à l'abattage),

- le mode de conduite (l'activité physique, la gestation et le vêlage), 
- la conduite alimentaire (la forme de la courbe de croissance, le niveau alimentaire, la nature des aliments, la durée de finition).

Nous plaçant dans un contexte législatif européen, nous n'envisagerons en revanche pas l'influence des facteurs de croissance. Ce sujet a cependant fait l'objet d'un paragraphe dans la revue bibliographique de Geay et al (2002).

L'impact des principaux facteurs d'élevage sur la tendreté des viandes sera évalué à travers les résultats des études portant à la fois sur la tendreté et les propriétés rhéologiques des viandes. La tendreté de la viande est le plus souvent évaluée par la méthode directe qu'est l'évaluation sensorielle, malgré les contraintes qu'elle implique (nombre de juges, quantité de viande à tester, coût élevé, répétabilité moyenne). C'est la méthode de référence à laquelle les mesures de force de cisaillement et de compression sont comparées (Touraille 1981). La force de cisaillement mesurée avec l'appareil de Salé (1971) ou avec l'appareil de Warner (1928) correspond à la contrainte nécessaire pour faire passer une arête tranchante à travers un morceau de viande perpendiculairement aux fibres musculaires. Deux phases peuvent être distinguées. Dans la première phase de compression, les myofibrilles se déplacent latéralement sous l'effet de la pression. Puis, dans la seconde phase de cisaillement, seul le tissu conjonctif est concerné (Salé 1971). Ainsi, déterminée sur une viande crue maturée où la résistance des myofibrilles est négligeable relativement à celle du conjonctif, la force de cisaillement représente avec une bonne approximation la résistance des tissus conjonctifs. Cette force est donc l'expression de la dureté de base de la viande, dureté qui ne peut être réduite par l'allongement de la durée de maturation puisque le tissu conjonctif n'est pas affecté significativement par les transformations post mortem (Purslow 1994). La tendreté peut également être évaluée par des mesures de compression. Selon la configuration choisie, longitudinale, transversale ou axiale, la déformation appliquée et la déformation latérale de l'échantillon peuvent être perpendiculaires ou dans le sens des fibres musculaires (Lepetit 1988). Selon l'orientation de l'échantillon et le taux de compression, ce test permet ainsi de mesurer la contribution respective des myofibres et du tissu conjonctif à la tendreté de la viande (Lepetit et Culioli 1994). La mesure de la force de com- pression donne alors une indication sur le degré de maturation du morceau considéré. Enfin, il est admis que le tissu conjonctif musculaire héberge une grande part des lipides du muscle qui ont un effet positif sur la tendreté. Cependant, bien que positives, les corrélations entre la teneur en lipides intramusculaires et la tendreté ou la force de cisaillement de la viande se révèlent peu élevées (Dransfield et al 1984, Shackelford et al 1994, Fiems et al 2000, Renand et al 2001, Jeremiah et al 2003).

\section{1 / Effets des caractéris- tiques propres de l'animal sur la tendreté de la viande et la résistance mécanique des muscles}

Les facteurs race et catégorie d'animal (jeune bovin, bœuf, génisse, vache) sont sans nul doute ceux qui font le plus varier la tendreté des viandes. Néanmoins, pour chaque catégorie d'animal d'une race donnée, d'autres facteurs intrinsèques peuvent intervenir dans le déterminisme de la tendreté. Nous passerons ainsi en revue successivement l'effet du type morphologique et de l'âge à l'abattage.

\section{1 / Effet du type morpholo- gique}

En France, dans certaines races à viande comme la race Charolaise, certains taureaux font l'objet d'une sélection sur les aptitudes bouchères notamment pour améliorer le poids de carcasse, d'autres sont plutôt sélectionnés sur les qualités maternelles. Ces différentes orientations se traduisent par l'existence de types morphologiques différents chez les descendants. Il existe ainsi une variabilité morphologique au sein même d'une race, depuis des animaux de type «viande» bien conformés, jusqu'à des animaux de type «élevage» au développement squelettique plus important mais moins conformés musculairement. Il faut donc se demander si cette divergence des objectifs de sélection modifie les propriétés sensorielles des viandes. Nous avons donc étudié l'effet du type morphologique sur la tendreté des viandes d'animaux, en excluant le type culard.

Quatre publications portant sur la comparaison des types morphologiques des pères des animaux ont été recensées. Elles permettent de mettre en évi- dence que des viandes de génisses élevées dans des conditions identiques et issues de taureaux Charolais de conformation moyenne ou élevée ont en moyenne une tendreté, une acceptabilité et une force de cisaillement équivalentes (Maher et al 2004). De même, la qualité sensorielle des viandes de 28 taurillons Angus descendants de 6 taureaux différents est équivalente (Ockerman et al 1984). Des résultats allant dans le même sens ont été rapportés par Altarriba et al (2005) à propos des notes de tendreté et des valeurs de force de compression mesurées à 20 et $80 \%$ de déformation dans le cas des muscles longissimus thoracis de 125 mâles entiers sélectionnés selon leur poids à 210 jours et issus de 9 taureaux distincts de race Pirenaica. Enfin, les viandes de 44 bœufs Japanese Black issus de quatre taureaux sélectionnés selon leur potentiel de croissance, ont en moyenne des proportions de lipides intramusculaires différentes mais des valeurs de forces de cisaillement très voisines (Ozawa et al 2000). L'impact du type morphologique de l'ascendant paternel d'un animal ne semble ainsi pas avoir d'effet significatif sur la tendreté ou les propriétés rhéologiques des viandes de ses descendants.

Outre la comparaison des types morphologiques des pères des animaux, l'effet du type morphologique des animaux eux-mêmes a également été testé. Lorsque des bœufs croisés (race Anglaise $\mathrm{X}$ race Continentale) de bon gabarit sont comparés à des animaux de gabarit moyen présentant des viandes plus persillées, les propriétés sensorielles des muscles longissimus thoracis sont jugées équivalentes (Camfield et al 1997). De même, les muscles longissimus thoracis de bœufs et génisses ayant un faible, un moyen ou un bon gabarit ne présentent pas de différences significatives de tendreté ou de force de cisaillement (Baublits et al 2006). L'absence de lien entre le type morphologique des animaux et la tendreté des viandes était à prévoir dans la mesure ou à même état d'engraissement, il n'existe que peu de différences entre les propriétés sensorielles des viandes d'animaux de races différentes (Dransfield et al 2003).

\section{2 / Effet de l'âge de l'animal}

La tendreté de la viande évolue peu dans le jeune âge et a tendance à diminuer lorsque les animaux deviennent adultes. Cette conclusion n'est pas récente puisqu'elle a déjà été proposée d'une part par Hiner et Hankins (1950) qui ont comparé des veaux âgés de 
2,5 mois à des vaches âgées de 67 mois et d'autre part par Henrickson et Moore (1965) qui ont comparé des veaux âgés de 6 mois à des bœufs de 18 mois, des génisses de 42 mois et des vaches de 90 mois. Nous envisagerons successivement l'évolution de la tendreté de la viande avec l'augmentation de l'âge à l'abattage pour les mâles entiers et castrés puis pour les femelles (génisses et vaches).

\section{a) Cas des bovins mâles}

Entre les âges de 12 et 24 mois chez le mâle, la plupart des essais répertoriés ne met pas en évidence d'augmentation de la dureté de la viande par analyse sensorielle (tableau 1-A). Entre 12 et 15 mois, les muscles longissimus thoracis et semitendinosus de taurillons des races Charolaise, Simmental, White Cattle, Danish Red, Romagnola, Chianina, Hereford, Blonde d'Aquitaine et Limousine, ne présentent pas de différence de force de cisaillement (Liboriussen et al 1977). Cependant, chaque lot d'âge à l'abattage étant constitué d'animaux de différentes races, la variabilité intra-lot a probablement limité les possibilités de mise en évidence de différences significatives. De même, entre 12 et 24 mois, de nombreux essais ne mettent pas en évidence d'effet significatif de l'augmentation de l'âge à l'abattage sur la tendreté de la viande ni sur sa force de cisaillement (Garcia-de Siles et al 1982, Dikeman et al 1986, Haurez et Joulie 1994, Dransfield et al 2003). Entre 15 et 19 mois, dans certains cas, les forces de cisaillement peuvent être plus élevées d'environ $20 \%$ chez les animaux les plus âgés (15 vs 19 mois) (Renand et al 1997). Cet effet se re-trouve au niveau des coefficients de corrélation négatifs

Tableau 1-A. Effet de l'âge sur la tendreté et la force de cisaillement des muscles longissimus thoracis de mâles entiers (ME) et castrés $(M C)$.

Les modifications sont exprimées en \% des valeurs obtenues pour le témoin (animal le plus jeune).

Le niveau de signification est indiqué entre parenthèses.

\begin{tabular}{|c|c|c|c|c|}
\hline Références & Type d'animal et race & $\begin{array}{c}\text { Age à } \\
\text { l'abattage } \\
\text { (mois) }\end{array}$ & $\begin{array}{l}\text { Note de } \\
\text { tendreté }\end{array}$ & $\begin{array}{c}\text { Force de } \\
\text { cisaillement }\end{array}$ \\
\hline $\begin{array}{l}\text { Liboriussen et al } \\
1977\end{array}$ & ME (différentes races ${ }^{1}$ ) & $\begin{array}{l}12 \\
15\end{array}$ & & $\begin{array}{c}4,26 \mathrm{~kg} \\
+0,7 \%(\mathrm{~ns})\end{array}$ \\
\hline \multirow{2}{*}{$\begin{array}{l}\text { Haurez et Joulie } \\
1994\end{array}$} & ME (Charolais - essai 1) & $\begin{array}{l}16 \\
19 \\
22 \\
\end{array}$ & $\begin{aligned} & 51 / 100 \\
&+ 2,0 \% \text { (ns) } \\
&- 2,0 \% \text { (ns) } \\
&\end{aligned}$ & \\
\hline & ME (Charolais - essai 2) & $\begin{array}{l}16 \\
19 \\
22 \\
\end{array}$ & $\begin{array}{c}51 / 100 \\
-3,9 \% \text { (ns) } \\
-2,0 \% \text { (ns) } \\
\end{array}$ & \\
\hline $\begin{array}{c}\text { Renand et al } \\
1997\end{array}$ & ME (Charolais) & $\begin{array}{l}15 \\
19 \\
\end{array}$ & $\begin{array}{c}10,2 / 20 \\
-11,6 \% \text { (ns) } \\
\end{array}$ & $\begin{aligned} & 1,25 \mathrm{~kg} \\
&+ \mathbf{2 0 , 2} \%\left(^{*}\right) \\
&\end{aligned}$ \\
\hline \multirow{4}{*}{$\begin{array}{c}\text { Dransfield et al } \\
2003\end{array}$} & ME (Aubrac) & $\begin{array}{l}15 \\
19 \\
24 \\
\end{array}$ & $\begin{array}{c}6,0 / 10 \\
+10,0 \% \text { (ns) } \\
+1,7 \%(n s) \\
\end{array}$ & \\
\hline & ME (Charolais) & $\begin{array}{l}15 \\
19 \\
24 \\
\end{array}$ & $\begin{aligned} & 5,8 / 10 \\
&+ 1,7 \% \text { (ns) } \\
&+ 1,7 \%(\mathrm{~ns}) \\
&\end{aligned}$ & \\
\hline & ME (Limousin) & $\begin{array}{l}15 \\
19 \\
24 \\
\end{array}$ & $\begin{array}{c}6,4 / 10 \\
-17,2 \%(\mathrm{~ns}) \\
-4,7 \%(\mathrm{~ns}) \\
\end{array}$ & \\
\hline & ME (Salers) & $\begin{array}{l}15 \\
19 \\
24 \\
\end{array}$ & $\begin{array}{c}6,3 / 10 \\
-12,7 \% \text { (ns) } \\
-3,2 \% \text { (ns) } \\
\end{array}$ & \\
\hline $\begin{array}{c}\text { Dikeman et al } \\
1986\end{array}$ & ME et MC (Angus) & $\begin{array}{l}12 \\
15 \\
18 \\
24\end{array}$ & $\begin{array}{r}7,06 / 10 \\
+1,8 \%(n s) \\
+4,1 \%(n s) \\
-0,7 \%(n s)\end{array}$ & $\begin{array}{c}5,85 \mathrm{~kg} \\
+3,1 \% \text { (ns) } \\
+17,8 \% \text { (ns) } \\
+19,3 \% \text { (ns) }\end{array}$ \\
\hline $\begin{array}{c}\text { Garcia-de Siles et al } \\
1982\end{array}$ & MC (croisés $\left.{ }^{2}\right)$ & $\begin{array}{l}12 \\
13 \\
14 \\
15 \\
16 \\
17\end{array}$ & $\begin{aligned} & 5,70 / 9 \\
+ & 1,6 \% \text { (ns) } \\
+ & 0,5 \% \text { (ns) } \\
+ & 2,1 \% \text { (ns) } \\
+ & 4,7 \% \text { (ns) } \\
+ & 0,0 \% \text { (ns) }\end{aligned}$ & $\begin{array}{c}7,99 \mathrm{~kg} \\
+0,4 \% \text { (ns) } \\
-3,0 \% \text { (ns) } \\
-6,9 \%(n s) \\
-3,3 \%(n s) \\
-6,9 \% \text { (ns) }\end{array}$ \\
\hline \multirow[t]{2}{*}{ Touraille 1982} & ME (Limousin) & $\begin{array}{l}16 \\
24 \\
26 \\
29\end{array}$ & $\begin{aligned} & 5,7 / 10 \\
+ & 7,7 \% \text { (ns) } \\
+ & 4,6 \% \text { (ns) } \\
+ & 6,2 \% \text { (ns) }\end{aligned}$ & \\
\hline & MC (Limousin) & $\begin{array}{l}33 \\
24 \\
33 \\
\end{array}$ & $\begin{array}{c}-16,9 \%\left(^{* * *}\right) \\
6,4 / 10 \\
-14,1 \%\left(^{* * *}\right)\end{array}$ & \\
\hline
\end{tabular}

ns : écart non significatif ; ${ }^{*}: p<0,05 ;{ }^{* * *}: p<0,001$.

1 Races testées: Charolaise, Simmental, White Cattle, Danish Red, Romagnola, Chianina, Hereford, Blonde d'Aquitaine et Limousine.

2 Animaux issus de croisement entre des vaches Angus-Holstein et des taureaux Angus-Hereford-Shorthorn. 
mis en évidence entre l'âge à l'abattage des taurillons et les notes de tendreté initiale $(-0,26)$ et de tendreté globale $(-0,29)$ de la viande, pour des taurillons Charolais abattus entre 16 et 21 mois (Renand et al 2001). Ce possible effet négatif de l'âge sur la tendreté va dans le même sens que l'augmentation de la teneur en collagène total du muscle et la diminution parallèle de la teneur en collagène soluble pour des animaux de 8 à 13 mois (Renand et al 1997), ou encore de 12 à 15 mois (Liboriussen et al 1977).
Au-delà de l'âge de 2 ans, il peut arriver que la tendreté se dégrade, comme le montre Touraille (1982) chez des taurillons Limousins de 33 mois vs 16 mois, de même que chez le bœuf entre 33 et 24 mois. Cette conclusion sur l'effet d'un âge élevé mérite cependant d'être confirmée, dans la mesure où la race et la précocité des animaux ont probablement un effet sur l'évolution de la tendreté de la viande avec l'âge. De plus, la difficulté à trouver des références bibliographiques sur l'effet de l'âge à l'abattage sur la quali- té sensorielle des viandes de bœufs traditionnels âgés, ne permet pas de conclure sur cette question de manière satisfaisante.

\section{b) Cas des femelles}

Entre 12 et 35 mois, la tendreté et/ou la force de cisaillement ne sont pas altérées par l'augmentation de l'âge à l'abattage (tableau 1-B). Le nombre de références sur le sujet étant limité, ces résultats restent encore à confirmer. Cependant, l'absence d'effet de l'âge à

Tableau 1-B. Effet de l'âge sur la tendreté et la force de cisaillement des muscles longissimus thoracis de femelles (F). Les modifications sont exprimées en \% des valeurs obtenues pour le témoin (animal le plus jeune).

Le niveau de signification est indiqué entre parenthèses.

\begin{tabular}{|c|c|c|c|c|}
\hline Références & Type d'animal et race & $\begin{array}{c}\text { Age à } \\
\text { l'abattage } \\
\text { (mois) }\end{array}$ & $\begin{array}{l}\text { Note de } \\
\text { tendreté }\end{array}$ & $\begin{array}{c}\text { Force de } \\
\text { cisaillement }\end{array}$ \\
\hline $\begin{array}{c}\text { Waggoner et al } \\
1990\end{array}$ & $\begin{array}{c}\text { F (Simmental } \\
\text { x Hereford) }\end{array}$ & $\begin{array}{l}17 \\
29 \\
\end{array}$ & $\begin{array}{c}6,5 / 8 \\
-7,7 \% \text { (ns) } \\
\end{array}$ & $\begin{array}{c}3,1 \mathrm{~kg} \\
+6,5 \% \text { (ns) } \\
\end{array}$ \\
\hline $\begin{array}{c}\text { Garcia-de Siles et al } \\
1982\end{array}$ & $\mathrm{~F}$ (croisés $\left.{ }^{2}\right)$ & $\begin{array}{l}12 \\
13 \\
14 \\
15 \\
16 \\
17 \\
18\end{array}$ & $\begin{array}{c}5,9 / 9 \\
-9,3 \% \text { (ns) } \\
-3,2 \% \text { (ns) } \\
+4,2 \% \text { (ns) } \\
+3,9 \% \text { (ns) } \\
-7,3 \% \text { (ns) } \\
+5,9 \% \text { (ns) }\end{array}$ & $\begin{aligned} & 8,75 \mathrm{~kg} \\
+ & 1,0 \%(\mathrm{~ns}) \\
+ & 0,7 \%(\mathrm{~ns}) \\
- & 0,8 \%(\mathrm{~ns}) \\
- & 5,7 \%(\mathrm{~ns}) \\
+ & 4,0 \%(\mathrm{~ns}) \\
- & 2,5 \%(\mathrm{~ns})\end{aligned}$ \\
\hline Touraille 1982 & $F($ Limousin) & $\begin{array}{l}24 \\
30 \\
\end{array}$ & $\begin{array}{c}7,0 / 10 \\
-8,6 \%(n s) \\
\end{array}$ & \\
\hline Field et al 1996 & F (Angus et Gelbvieh) & $\begin{array}{l}31 \\
33 \\
35\end{array}$ & $\begin{array}{c}6,33 / 12 \\
-6,5 \%(n s) \\
+10,1 \%(n s)\end{array}$ & $\begin{array}{c}9,14 \mathrm{~kg} \\
+4,8 \% \text { (ns) } \\
+0,0 \% \text { (ns) }\end{array}$ \\
\hline \multirow{4}{*}{$\begin{array}{l}\text { Dransfield et al } \\
2003\end{array}$} & F (Aubrac) & $\begin{array}{c}60 \\
78 \\
102 \\
\end{array}$ & $\begin{array}{c}7,1 / 10 \\
-14,1 \% \text { (ns) } \\
-7,0 \% \text { (ns) }\end{array}$ & \\
\hline & F (Charolais) & $\begin{array}{c}60 \\
78 \\
102 \\
\end{array}$ & $\begin{array}{c}5,9 / 10 \\
-3,4 \% \text { (ns) } \\
+13,6 \% \text { (ns) } \\
\end{array}$ & \\
\hline & F (Limousin) & $\begin{array}{c}60 \\
78 \\
102 \\
\end{array}$ & $\begin{array}{c}6,5 / 10 \\
-3,1 \% \text { (ns) } \\
-4,6 \% \text { (ns) } \\
\end{array}$ & \\
\hline & F (Salers) & $\begin{array}{c}60 \\
78 \\
102 \\
\end{array}$ & $\begin{aligned} & 6,3 / 10 \\
&+ 6,3 \%(n s) \\
&- 17,5 \%\left(^{*}\right) \\
&\end{aligned}$ & \\
\hline Dumont et al 1991 & $\mathrm{~F}$ (Charolais) & $\begin{array}{c}60 \\
132 \\
\end{array}$ & $\begin{array}{c}5,85 / 10 \\
-9,4 \%\left(^{*}\right) \\
\end{array}$ & $\begin{array}{c}1,65 \mathrm{~kg} \\
-1,2 \% \text { (ns) } \\
\end{array}$ \\
\hline \multirow{2}{*}{ Bastien et al 2002} & F (Normande) & $\begin{array}{c}60 \\
132 \\
\end{array}$ & $\begin{array}{r}59,0 / 100 \\
+8,6 \%(n s)\end{array}$ & \\
\hline & F (Limousin) & $\begin{array}{c}60 \\
132 \\
\end{array}$ & $\begin{array}{c}70,0 / 100 \\
+0,0 \% \text { (ns) } \\
\end{array}$ & \\
\hline \multirow{2}{*}{ Muller et al 1992} & F (Charolais) & $\begin{array}{c}78 \\
102 \\
108 \\
\end{array}$ & $\begin{array}{r}5,43 / 9 \\
+3,3 \% \text { (ns) } \\
-4,1 \%(n s) \\
\end{array}$ & $\begin{aligned} & 7,10 \mathrm{~kg} \\
&+ 7,6 \% \text { (ns) } \\
&+ 4,4 \% \text { (ns) } \\
&\end{aligned}$ \\
\hline & $\mathrm{F}$ (Angus) & $\begin{array}{c}78 \\
102 \\
108 \\
\end{array}$ & $\begin{array}{c}5,50 / 9 \\
-1,6 \% \text { (ns) } \\
-5,5 \% \text { (ns) } \\
\end{array}$ & $\begin{array}{c}+7,86 \mathrm{~kg} \\
-1,8 \% \text { (ns) } \\
+27,1 \%\left(^{*}\right)\end{array}$ \\
\hline Shemeis et al 1994 & F (Danish Friesian) & $\begin{array}{c}90 \\
112 \\
135\end{array}$ & & $\begin{array}{c}5,99 \mathrm{~kg} \\
+16,2 \%\left(^{*}\right) \\
+15,4 \%\left(^{*}\right)\end{array}$ \\
\hline
\end{tabular}

ns : écart non significatif ; ${ }^{*}: p<0,05 ;{ }^{* * *}: p<0,001$. 
l'abattage dans cette tranche d'âge, semble accrédité par des teneurs en collagène total et soluble voisines entre des viandes de génisses de 12 et 24 mois (Waggoner et al 1990) et de 31 à 35 mois (Field et al 1996). Lorsque les femelles sont abattues plus âgées, il n'y a pas de différence de tendreté entre groupes d'animaux d'âges différents pour un même état d'engraissement des carcasses ou une même teneur en lipides intramusculaires. Ainsi, la tendreté et/ou la force de cisaillement de la viande sont proches :

- entre la viande de vaches Limousines ou Normandes âgées de 3,5 à 5 ans ou de 9 à 11 ans que l'analyse porte sur le muscle longissimus thoracis ou sur le supraspinatus (Bastien et al 2002),

- entre la viande de vaches Charolaises de 6/7 ans, 8/9 ans ou de plus de 9 ans (Muller et al 1992).

Si la tendreté de la viande de vaches Angus n'est pas modifiée par l'âge, une augmentation de la force de cisaillement de $27,1 \%$ est observée entre des animaux de $6 / 7$ ans et $8 / 9$ ans et des animaux de plus de 9 ans (Muller et al 1992). Cette évolution se retrouve pour la force de cisaillement des viandes entre des vaches Danoises de moins de 3 ans, de 3 à 5 ans et de plus de 4/5 ans (Shemeis et al 1994). Cependant, l'effet âge sur la tendreté de la viande semble variable selon la race considérée (Liboriussen et al 1977). Pour exemple, lors de la comparaison de races différentes (Aubrac, Limousine, Salers et Charolaise) seule la tendreté du muscle triceps brachii des animaux Salers est altérée par l'augmentation de l'âge (entre 4/5 ans, 6/7 ans et 8/9 ans, Dransfield et al 2003).

Dans certains cas, en exacerbant l'écart entre âges moyens, par exemple 5 et 11 ans chez la vache de race Charolaise, on peut arriver à mettre en évidence une dégradation d'environ $10 \%$ de la note de tendreté. A même durée de maturation de 14 jours, la tendreté des muscles longissimus thoracis des vaches de 11 ans est notée significativement inférieure à celle de vaches de 5 ans (Dumont et al 1991). Cette évolution ne se retrouvant ni sur la force de cisaillement, ni sur la teneur en collagène total des muscles longissimus thoracis et triceps brachii, il faut se demander si l'âge intervient en freinant le processus de maturation, et notamment sa vitesse.

\section{2 / Effet du mode de conduite sur la tendreté de la viande et la résistance mécanique des muscles}

Au sein des facteurs relatifs au mode de conduite, nous passerons en revue successivement les effets de l'activité physique et de l'état physiologique des femelles.

\section{1 / Effet de l'activité physique de l'animal}

L'impact de l'activité physique sur la tendreté de la viande a été étudié en bibliographie à travers la comparaison de conduites à l'auge ou à la pâture ou encore de conduites en stabulation libre ou en stabulation entravée. L'exercice n'affecte pas les propriétés rhéologiques des muscles non locomoteurs. Ainsi, la force de compression à 20 et $80 \%$ de déformation est équivalente entre les viandes de génisses finies en stabulation libre ou en stabulation entravée que l'analyse concerne le muscle longissimus thoracis ou le muscle rectus abdominis (Berge et al 1991). De même, entre des animaux finis à l'auge ou à la pâture, on n'observe pas de différence de force de cisaillement du muscle longissimus thoracis (Moloney et al 2004).

L'exercice semble en revanche affecter les propriétés sensorielles et rhéologiques des muscles locomoteurs. Ainsi, la conduite de taurillons en stabulation libre comparée à l'élevage en stabulation entravée est à l'origine d'une diminution de l'ordre de $30 \%$ de la force de cisaillement du muscle semitendinosus (Lahucky et al 1998). De même, des résultats antérieurs portant sur des moutons ont permis de mettre en évidence que les muscles semimembranosus des animaux faisant de l'exercice étaient potentiellement plus tendres que ceux des animaux sans activité physique (Aalhus et al 1991).

\section{2 / Effet de la gestation et du vêlage}

Lorsque les femelles sont abattues à même âge (tableau 2), les génisses ont à l'abattage un poids de carcasse significativement supérieur aux primipares (Waggoner et al 1990, Field et al 1996), excepté dans le cas où les âges et poids à l'abattage sont fixés dans le protocole (Dumont et al 1987, Micol et al 1992).
Deux essais permettent de conclure que la gestation et le vêlage ne modifient pas de façon significative la tendreté de la viande, ni les forces de cisaillement et de compression du muscle (Micol et al 1992, Field et al 1996).

Dans certains cas, le vêlage peut s'accompagner d'une diminution de la force de cisaillement. Ainsi, dans une étude portant sur 111 femelles Simmental $x$ Hereford, la force de cisaillement des viandes de primipares de 29 mois a été inférieure d'environ $8 \%$ à celle de génisses de même âge pour le muscle longissimus thoracis (Waggoner et al 1990), sans répercution sur les notations de tendreté.

Les écarts observés et leurs sens ne sont pas toujours faciles à interpréter car ils semblent dépendre des méthodes d'estimation (évaluation sensorielle ou force de cisaillement), du mode de présentation des échantillons (cuit ou cru), du type de muscle proposé mais aussi des autres facteurs d'élevage susceptibles d'interagir (type génétique, niveau d'alimentation). Ainsi, dans une étude menée sur 7 muscles de 158 femelles de races Holstein et Charolais $x$ Holstein, il a été observé (Dumont et al 1987) :

- des tendretés ou des forces de cisaillement équivalentes entre des génisses n'ayant pas vêlé et des primipares de même âge pour les muscles triceps brachii, adductor et serratus ventralis thoracis,

- une différence de force de cisaillement et/ou de tendreté en faveur des génisses pour les muscles longissimus thoracis, pectoralis ascendens et trapezius,

- une différence de tendreté en faveur des primipares et une différence de force de cisaillement en faveur des génisses pour le muscle semitendinosus.

Lorsque les femelles qui ont vêlé sont abattues à un âge et un poids supérieurs à celui des nullipares (tableau 2), il n'apparaît pas d'effet significatif de la gestation et du vêlage sur la tendreté et la force de cisaillement de la viande dans la majorité des essais (Shackelford et al 1995, Fiems et al 2003, Cabaraux et al 2004). Mais, dans certains cas, la viande de génisse a des propriétés sensorielles plus favorables que la viande des femelles qui ont vêlé. Ainsi, lors de la comparaison de génisses de 18 mois et de vaches de 34 à 80 mois, la viande des génisses nécessite des forces de cisaillement significativement inférieures à celle des 
Tableau 2. Effet de la gestation et du vêlage sur les teneurs en lipides intramusculaires musculaires et sur les propriétés rhéologiques et sensorielles de la viande.

Les modifications sont exprimées en \% des valeurs obtenues pour le témoin (génisse). Le niveau de signification est indiqué entre parenthèses.

\begin{tabular}{|c|c|c|c|c|c|c|c|}
\hline Références & Age (mois) & Muscle & $\begin{array}{l}\text { Note de } \\
\text { tendreté }\end{array}$ & $\begin{array}{l}\text { Teneur en } \\
\text { Lipides } \\
\text { totaux }\end{array}$ & $\begin{array}{l}\text { Force de } \\
\text { Cisaillement } \\
\text { (viande } \\
\text { crue) }\end{array}$ & $\begin{array}{l}\text { Force de } \\
\text { cisaillement } \\
\text { (viande } \\
\text { cuite) }\end{array}$ & $\begin{array}{l}\text { Force de } \\
\text { compression }\end{array}$ \\
\hline $\begin{array}{c}\text { Dumont et al } \\
1987\end{array}$ & $\begin{array}{l}\text { Génisse - } 23 \text { mois } \\
\text { Primipare }-23 \text { mois } \\
\text { Génisse }-23 \text { mois } \\
\text { Primipare }-23 \text { mois } \\
\text { Génisse }-23 \text { mois } \\
\text { Primipare }-23 \text { mois } \\
\text { Génisse }-23 \text { mois } \\
\text { Primipare }-23 \text { mois } \\
\text { Génisse }-23 \text { mois } \\
\text { Primipare - } 23 \text { mois } \\
\text { Génisse }-23 \text { mois } \\
\text { Primipare }-23 \text { mois } \\
\text { Génisse }-23 \text { mois } \\
\text { Primipare }-23 \text { mois }\end{array}$ & $\begin{array}{l}\text { LT } \\
\text { ST } \\
\text { AD } \\
\text { TB } \\
\text { SVT } \\
\text { PA } \\
\text { TP }\end{array}$ & $\begin{array}{c}137 / 320^{1} \\
+24,7 \%\left(^{*}\right) \\
180 / 320^{1} \\
-28,6 \%\left(^{*}\right) \\
170 / 320^{1} \\
-11,8 \%(\mathrm{~ns})\end{array}$ & & $\begin{array}{c}1,68 \mathrm{~kg} \\
\left.-6,1 \% \mathrm{(+}^{+}\right) \\
5,10 \mathrm{~kg} \\
-11 \%\left(^{* * *}\right) \\
1, \mathrm{N58} \mathrm{kg} \\
-7,1 \%(\mathrm{~ns}) \\
2,89 \mathrm{~kg} \\
-6,3 \%(\mathrm{~ns}) \\
3,27 \mathrm{~kg} \\
-3,4 \%(\mathrm{~ns}) \\
6,11 \mathrm{~kg} \\
-11,4 \%\left(^{*}\right) \\
6,39 \mathrm{~kg} \\
-, 9 \%\left(^{+}\right) \\
\end{array}$ & $\begin{array}{c}5,46 \mathrm{~kg} \\
-7,5 \%\left(^{*}\right) \\
2,51 \mathrm{~kg} \\
-0,4 \%(\mathrm{~ns}) \\
3,65 \mathrm{~kg} \\
-7,3 \%(\mathrm{~ns}) \\
3,75 \mathrm{~kg} \\
+0,5 \%(\mathrm{~ns}) \\
4,37 \mathrm{~kg} \\
-1,4 \%(\mathrm{~ns}) \\
4,27 \mathrm{~kg} \\
-3,4 \%(\mathrm{~ns})\end{array}$ & \\
\hline $\begin{array}{l}\text { Micol et al } \\
1992\end{array}$ & $\begin{array}{l}\text { Génisse - } 36 \text { mois } \\
\text { Primipare - } 36 \text { mois } \\
\text { Génisse - } 36 \text { mois } \\
\text { Primipare - } 36 \text { mois }\end{array}$ & $\begin{array}{l}\text { LT } \\
\text { TB }\end{array}$ & & & & & $\begin{array}{c}1,42 \mathrm{~kg} \\
-14,3 \% \text { (ns) } \\
1,83 \mathrm{~kg} \\
+11,1 \% \text { (ns) }\end{array}$ \\
\hline $\begin{array}{l}\text { Waggoner et al } \\
1990\end{array}$ & $\begin{array}{l}\text { Génisse - } 29 \text { mois } \\
\text { Primipare - } 29 \text { mois } \\
\text { (abattue plus lourde) }\end{array}$ & $\mathrm{LT}$ & $\begin{array}{c}5,6 / 8 \\
+7,1 \%(\mathrm{~ns})\end{array}$ & & $\begin{array}{c}3,6 \mathrm{~kg} \\
-8,3 \%\left(^{*}\right)\end{array}$ & & \\
\hline $\begin{array}{l}\text { Field et al } \\
1996\end{array}$ & $\begin{array}{l}\text { Génisse - } 33 \text { mois } \\
\text { Primipare - } 33 \text { mois } \\
\text { (abattue plus lourde) }\end{array}$ & & $\begin{array}{c}6,60 / 12 \\
-4,2 \%(n s)\end{array}$ & & $\begin{array}{c}9,29 \mathrm{~kg} \\
+3,0 \%(\mathrm{~ns})\end{array}$ & & \\
\hline $\begin{array}{c}\text { Shackelford et al } \\
1995\end{array}$ & $\begin{array}{c}\text { Génisse }-22 \text { mois } \\
\text { Primipare }-35 \text { mois }\end{array}$ & LT & $\begin{array}{c}5,0 / 8 \\
-8,0 \%(n s) \\
\end{array}$ & & $\begin{array}{c}6,0 \mathrm{~kg} \\
+1,7 \% \text { (ns) }\end{array}$ & & \\
\hline $\begin{array}{l}\text { Patterson et al } \\
2002\end{array}$ & $\begin{array}{l}\text { Génisse - } 18 \text { mois } \\
\text { Primipare }-34 \text { mois } \\
\text { Vache ayant vêlé } \\
3 \text { fois }-55 \text { mois } \\
\text { Vache ayant vêlé } \\
5 \text { fois }-80 \text { mois }\end{array}$ & LT & & & $\begin{array}{c}3,10 \mathrm{~kg} \\
+32,6 \%\left(^{* * *}\right) \\
+45,7 \%\left(^{* * *}\right) \\
+54,3 \%\left(^{* * *}\right)\end{array}$ & & \\
\hline $\begin{array}{l}\text { Fiems et al } \\
2003\end{array}$ & $\begin{array}{c}\text { Génisse - } 28 \text { mois } \\
\text { Primipare - } 40 \text { mois } \\
\text { Vache ayant vêlé } \\
2 \text { fois - } 49 \text { mois } \\
\text { Vache ayant vêlé } \\
3 \text { fois - } 62 \text { mois } \\
\text { Vache ayant vêlé } \\
4 \text { fois - } 75 \text { mois } \\
\text { Vache ayant vêlé } \\
5 \text { fois - } 88 \text { mois } \\
\text { Vache ayant vêlé } \\
6 \text { fois } \\
\text { ou plus - } 111 \text { mois }\end{array}$ & LT & & $\begin{array}{c}2,8 \% \\
-25,0 \%\left(^{*}\right) \\
-28,6 \%\left(^{*}\right) \\
-10,7 \% \\
(n s) \\
-25,0 \% \\
\text { (ns) } \\
-10,7 \% \\
\text { (ns) } \\
-28,6 \%\left(^{*}\right)\end{array}$ & $\begin{array}{c}3,62 \mathrm{~kg} \\
-4,5 \%(n s) \\
-4,8 \%(n s) \\
-7,3 \%(n s) \\
-19,4 \%(n s) \\
-19,7 \%(n s) \\
-16,1 \%(n s)\end{array}$ & & \\
\hline $\begin{array}{c}\text { Cabaraux et al } \\
2004\end{array}$ & $\begin{array}{l}\text { Génisse - } 34 \text { mois } \\
\text { Vache ayant vêlé } \\
2 \text { fois - } 57 \text { mois } \\
\text { Vache ayant vêlé } \\
4 \text { fois- } 90 \text { mois }\end{array}$ & LT & & $\begin{array}{c}5,8 \% \mathrm{MS} \\
+34,5 \% \\
\left({ }^{* * *}\right) \\
-19,0 \% \\
(\mathrm{~ns})\end{array}$ & $\begin{array}{c}3,47 \\
-10,9 \% \text { (ns) } \\
-7,4 \%(n s)\end{array}$ & & \\
\hline
\end{tabular}

ns : différence non significative ; $+: p<0,10 ;{ }^{*}: p<0,05 ;{ }^{* *}: p<0,01 ; * * * p<0,001$.

LT : longissimus thoracis ; TP : trapezius; PA : pectoralis ascendens ; SVT : serratus ventralis thoracis ; TB : triceps brachii ; AD : adductor ; ST : semitendinosus ; RA : rectus abdominis.

1 La tendreté a été estimée en comptant pour chaque type d'animal le nombre d'échantillons estimés comme les plus tendres. 
animaux qui ont vêlé (Patterson et al 2002). Il est ainsi possible de supposer que la gestation et le vêlage ont un effet négatif sur la tendreté de la viande. Cet effet négatif semble exacerbé par un abattage des vaches à un âge supérieur en comparaison des génisses. L'accumulation des effets négatifs de l'âge à l'abattage et du facteur gestation et vêlage pourrait ainsi jouer en défaveur des viandes de vaches.

\section{3 / Effet de la conduite ali- mentaire sur la tendreté de la viande et la résistance mécanique des muscles}

Pour étudier l'influence des facteurs alimentaires sur la tendreté de la viande, on peut s'intéresser à l'ensemble de la vie de l'animal ou plus spécifiquement à la période de finition. Peu de travaux sont disponibles sur l'effet de la forme globale de la courbe de croissance à l'exception de ceux qui ont tenté de mesurer l'effet de la croissance compensatrice. Ces essais portent généralement sur la viande de taurillons, de bœufs et/ou de génisses mais rarement sur celle de vaches adultes.

Dans ce paragraphe, nous aborderons successivement l'effet de la forme de la courbe de croissance, du niveau alimentaire, de la nature de l'alimentation et de la durée de finition sur la tendreté et les mesures rhéologiques.

\section{1 / Effet de la forme de la cour- be de croissance}

L'éleveur peut faire subir à l'animal des périodes de restriction alimentaire qui provoquent un ralentissement de la croissance. Lors du retour à une alimentation non limitée, il peut s'ensuivre une phase d'accroissement du gain de poids des animaux par rapport à la normale. L'alternance, volontaire ou non, de périodes d'apports alimentaires réduits puis élevés engendre chez les bovins un phénomène de croissance compensatrice. Une restriction telle que le poids de l'animal augmente très lentement ou stagne voire diminue pendant une durée suffisante, rend possible l'adaptation de l'animal à cet état de faible nutrition (Ryan 1990). Après cette période de restriction alimentaire, le retour à une alimentation non limitée peut conduire à une phase de compensation. La croissance compensatrice se traduit alors par un accroissement du gain de poids des animaux par rapport à la normale. Il existe assez peu de résul- tats sur l'effet de la courbe de croissance sur la tendreté (Hoch et al 2003). Selon Tatum (1981), la croissance compensatrice serait bénéfique pour les propriétés sensorielles de la viande mais la plupart des travaux plus récents ne mettent en évidence que peu d'effet significatif de la croissance compensatrice sur la tendreté ou les propriétés rhéologiques de la viande (tableau 3).

Après une restriction de 30 jours des apports d'aliments concentrés, chez le taurillon, la mise à disposition d'aliments concentrés à volonté ne modifie pas la tendreté ou la note globale d'appréciation de la viande (Cerdeno et al 2006). Dans cet essai, la mise à disposition d'aliments concentrés ad libitum n'a pas induit de véritable compensation des taurillons $(1,3 \mathrm{~g} / \mathrm{j}$ vs $1,5 \mathrm{~g} / \mathrm{j})$, ce qui peut expliquer en partie l'absence d'effet significatif sur la tendreté de la viande. Selon les auteurs, l'absence d'effet significatif peut également être attribuée à la courte durée de la phase de compensation (30 jours), une période minimale de 95 jours étant nécessaire pour observer un effet significatif de la compensation sur la force de cisaillement.

Après une restriction de 112 jours chez le bœuf, la croissance compensatrice n'a pas d'influence sur la tendreté et les propriétés rhéologiques du muscle longissimus thoracis mais le muscle semitendinosus des animaux restreints puis compensés est $10 \%$ plus tendre que celui des animaux témoins, mettant ainsi en évidence un possible effet favorable de la croissance compensatrice pour un muscle de tendreté intermédiaire (Laborde et al 2002). Chez des bœufs plus légers à l'abattage, cet effet favorable de la croissance compensatrice sur la tendreté du muscle semitendinosus n'est cependant pas retrouvé après une restriction de 100 jours. Ainsi, la croissance compensatrice, réalisée à l'auge avec des concentrés ou à la pâture, ne modifie pas la force de cisaillement et les pertes de poids à la cuisson des muscles semitendinosus de bœufs (Allingham et al 1998).

Après une restriction de 115 jours, la croissance compensatrice n'a pas d'effet sur les valeurs de force de cisaillement du muscle longissimus thoracis de taurillons (Hornick et al 1998). Au delà, après une restriction de 239 jours, la croissance compensatrice semble pouvoir conduire à une diminution d'environ $12 \%$ de la force de cisaillement (Hornick et al 1998). Cet effet bénéfique de la croissance compensatrice n'apparaîtrait donc qu'au delà d'une certaine durée des phases de restriction et de compensation et n'existerait plus après une durée de restriction trop importante de 411 jours par exemple (Hornick et al 1998).

La diminution de la force de cisaillement de la viande sous l'effet de la croissance compensatrice peut s'expliquer par l'accélération du turnover des protéines, susceptible de conduire à une néo-synthèse de collagène (Kopp et Bonnet 1982, Crouse et al 1986). Une augmentation de la synthèse de collagène de solubilité plus élevée ou une réduction de la taille des fibres de collagène de type I pourrait également expliquer cette amélioration de la tendreté sous l'effet de la croissance compensatrice (Geay et al 2002). Enfin, l'accroissement de la tendreté pourrait être relié à l'accroissement de la proportion de fibres musculaires glycolytiques, à maturation plus rapide, aux dépens de la proportion de fibres lentes (Picard et al 1995).

De manière paradoxale, une croissance compensatrice pourrait s'accompagner d'une augmentation de la force de cisaillement d'environ $14 \%$ pour les bœufs restreints puis compensés selon Muir et al (2001). Ces auteurs mettent également en évidence sur les mêmes animaux une diminution de 14 et $23 \%$ respectivement de l'activité des $\mathrm{m}$ - et $\mu$-calpaïnes et une augmentation de $18,5 \%$ de celle des calpastatines dans les muscles des animaux restreints puis compensés. Selon les auteurs, la moindre activité du système protéolytique pourrait pour partie être attribuée à l'augmentation de 40 jours de l'âge à l'abattage des animaux restreints puis compensés.

\section{2 / Influence de l'augmenta- tion du niveau alimentaire}

Pour des animaux restreints durant l'hiver suivant le sevrage et abattus à un âge supérieur à 2 ans, le niveau alimentaire du premier hiver ne semble pas avoir d'effet significatif sur la tendreté de la viande. Par exemple, la force de cisaillement des muscles de génisses abattues à 36 mois n'est pas modifiée par l'augmentation du niveau alimentaire de la ration distribuée entre 6 et 15 mois (Wertz et al 2001). Le nombre d'essais sur le sujet étant très limité, la période d'élevage ne sera pas étudiée dans cette partie et seuls les effets des variations au cours de la période de finition seront développés. 
Tableau 3. Influence d'une période de croissance compensatrice sur la tendreté et les propriétés rhéologiques de la viande. Les modifications sont exprimées en \% des valeurs obtenues pour le témoin (animal en croissance continue). Le niveau de signification est indiqué entre parenthèses.

\begin{tabular}{|c|c|c|c|c|c|c|c|c|}
\hline 黑 & 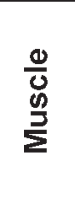 & 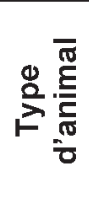 & Lots & 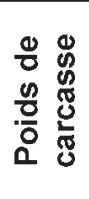 & $\begin{array}{l}\text { Gain de poids vif } \\
\text { des animaux témoins } \\
\text { (en rosé) et des } \\
\text { animaux restreints } \\
\text { puis compensés }(\mathrm{kg} / \mathrm{j})\end{array}$ & $\begin{array}{c}\text { Force } \\
\text { de } \\
\text { compression }\end{array}$ & $\begin{array}{c}\text { Force } \\
\text { de } \\
\text { cisaillement }\end{array}$ & $\begin{array}{c}\text { Note } \\
\text { de } \\
\text { tendreté }\end{array}$ \\
\hline $\begin{array}{c}\text { Allingham } \\
\text { et al } \\
1998\end{array}$ & ST & B & $\begin{array}{c}\text { Témoin en } \\
\text { croissance } \\
\text { continue } \\
100 \mathrm{j} \text { de restriction } \\
157 \mathrm{j} \text { de compensation } \\
100 \mathrm{j} \text { de restriction } \\
157 \mathrm{j} \text { de compensation }\end{array}$ & $\begin{array}{l}200 \\
171 \\
218\end{array}$ & & $\begin{array}{c}2,6 \mathrm{~kg} \\
-7,7 \% \text { (ns) } \\
-19,2 \%(n s)\end{array}$ & $\begin{array}{r}4,6 \mathrm{~kg} \\
+4,3 \%(\mathrm{~ns}) \\
+0,0 \%(\mathrm{~ns})\end{array}$ & \\
\hline $\begin{array}{l}\text { Hornick } \\
\text { et al } \\
1998\end{array}$ & LT & $\mathrm{T}$ & $\begin{array}{l}\text { Témoin en croissance } \\
\text { continue } \\
115 \mathrm{j} \text { de restriction } \\
147 \mathrm{j} \text { de compensation } \\
239 \mathrm{j} \text { de restriction } \\
120 \mathrm{j} \text { de compensation } \\
411 \mathrm{j} \text { de restriction } \\
112 \mathrm{j} \text { de compensation }\end{array}$ & $\begin{array}{l}399 \\
390 \\
396 \\
447\end{array}$ & $\begin{array}{c}1,3 \\
0,6-1,5 \\
0,6-1,4 \\
0,4-1,5\end{array}$ & & 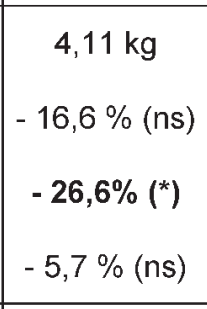 & \\
\hline $\begin{array}{l}\text { Muir } \\
\text { et al } \\
2001\end{array}$ & LT & B & $\begin{array}{c}\text { Témoin en croissance } \\
\text { continue } \\
126 \mathrm{j} \text { de restriction } \\
153 \mathrm{j} \text { de compensation }\end{array}$ & $\begin{array}{l}316 \\
311\end{array}$ & $\begin{array}{c}0,7 \\
0,1-0,9\end{array}$ & & $\begin{array}{c}5,49 \mathrm{~kg} \\
+13,5 \% \\
\left(\begin{array}{l}1 * \star * \\
*\end{array}\right.\end{array}$ & \\
\hline \multirow{2}{*}{$\begin{array}{l}\text { Laborde } \\
\text { et al } \\
2002\end{array}$} & LT & B & \multirow{2}{*}{$\begin{array}{c}\text { Témoin en croissance } \\
\text { continue } \\
112 \mathrm{j} \text { de restriction } \\
112 \mathrm{j} \text { de compensation } \\
\text { Témoin en croissance } \\
\text { continue } \\
112 \mathrm{j} \text { de restriction } \\
112 \mathrm{j} \text { de compensation }\end{array}$} & $\begin{array}{l}360 \\
348\end{array}$ & $\begin{array}{c}1,9 \\
0,5-2,2\end{array}$ & & $\begin{array}{c}5,83 \mathrm{~kg} \\
-3,8 \%(\mathrm{~ns})\end{array}$ & $\begin{array}{c}6,89 / 15 \\
+4,5 \% \\
\text { (ns) }\end{array}$ \\
\hline & ST & B & & $\begin{array}{l}360 \\
348\end{array}$ & $\begin{array}{c}1,9 \\
0,5-2,2\end{array}$ & & $\begin{array}{c}6,24 \mathrm{~kg} \\
+0,0 \%(\mathrm{~ns})\end{array}$ & $\begin{array}{c}7,74 / 15 \\
+9,9 \% \\
(* *) \\
\end{array}$ \\
\hline \multirow{2}{*}{$\begin{array}{l}\text { Cerdeno } \\
\text { et al } \\
2006\end{array}$} & LT & $\mathrm{T}$ & \multirow{2}{*}{$\begin{array}{c}\text { Témoin en croissance } \\
\text { continue } \\
30 \mathrm{j} \text { de restriction } \\
30 \mathrm{j} \text { de compensation } \\
\text { Témoin en croissance } \\
\text { continue } \\
30 \mathrm{j} \text { de restriction } \\
30 \mathrm{j} \text { de compensation }\end{array}$} & $\begin{array}{l}232 \\
212\end{array}$ & $\begin{array}{c}1,5 \\
1,3-1,2\end{array}$ & & $\begin{array}{c}5,7 \mathrm{~kg} \\
-8,8 \%(n s)\end{array}$ & $\begin{array}{r}6,0 / 10 \\
+15,0 \% \\
\text { (ns) }\end{array}$ \\
\hline & LT & $\mathrm{T}$ & & $\begin{array}{l}232 \\
212\end{array}$ & $\begin{array}{c}1,9 \\
1,3-1,2\end{array}$ & & $\begin{array}{c}4,8 \mathrm{~kg} \\
+8,3 \%(\mathrm{~ns})\end{array}$ & $\begin{array}{c}6,0 / 10 \\
+15,0 \% \\
(\mathrm{~ns})\end{array}$ \\
\hline
\end{tabular}

$\mathrm{T}$ : taurillon, B : bœuf.

ns : écart non significatif $;+: p<0,10 ;{ }^{* *}: p<0,01 ;{ }^{* * *}: p<0,001$.

LT : longissimus thoracis; ST : semitendinosus.

a) Augmentation du niveau alimentaire en finition sans modification de la nature de la ration

Les dispositifs expérimentaux portant sur l'étude de l'effet du niveau alimentaire en finition sont organisés de façon à ce que les animaux soient abattus soit à même âge avec des poids variables, soit à même poids mais à des âges différents (tableau 4). Lorsque les animaux sont abattus à même âge, la réduction du niveau alimentaire de la ration entraîne une diminution du poids de carcasse des animaux. Lorsque les animaux sont abattus à même poids, la croissance des animaux alimentés en niveau bas est compensée par une augmentation de la durée de finition et l'ef- fet mis en évidence résulte donc de l'interaction des effets du niveau alimentaire de la ration et de la durée de finition voire de l'âge de l'animal.

- Effet du niveau alimentaire lors d'un abattage à même âge

Quel que le soit le type d'animal considéré (bœuf, génisse, taurillon, vache), les différents essais ne mettent pas en évidence d'effet significatif de l'augmentation du niveau alimentaire de la ration de finition sur les notes de tendreté malgré l'élévation de la teneur en lipides intramusculaires dans la plupart des essais (Burson et al 1980, Gregory et al 1994, Sinclair et al 2001, Frickh et al 2003, Sami et al 2004,
Marino et al 2006). De manière analogue, Hennessy et Morris (2003) n'observent pas de différence de qualité globale de la viande, descripteur synthétique regroupant la tendreté, la jutosité et la flaveur de la viande. De même, Cepin et al (1992) n'observent pas de corrélation significative entre le gain moyen quotidien des animaux en finition et la tendreté de la viande ou la force de cisaillement.

La force de cisaillement n'est généralement pas modifiée par l'augmentation du niveau alimentaire de la ration en finition (Burson et al 1980, Roux et al 1993, Sinclair et al 2001, Frickh et al 2003, Cooke et al 2004, Sami et al 
Tableau 4. Influence de l'augmentation du niveau alimentaire en finition sur la tendreté, la force de cisaillement et la teneur en lipides intramusculaires de la viande.

Les modifications sont exprimées en \% des valeurs obtenues pour le témoin (niveau le plus faible).

Le niveau de signification est indiqué entre parenthèses.

\begin{tabular}{|c|c|c|c|c|c|c|c|}
\hline Références & $\begin{array}{c}\text { Type } \\
\text { d'animal }\end{array}$ & Muscle & $\begin{array}{c}\text { Âge } \\
\text { (mois) }\end{array}$ & $\begin{array}{c}\text { Gain au } \\
\text { Cours de } \\
\text { la finition } \\
(\mathrm{kg} / \mathrm{j})\end{array}$ & $\begin{array}{l}\text { Note de } \\
\text { tendreté }\end{array}$ & $\begin{array}{l}\text { Teneur en } \\
\text { lipides } \\
\text { totaux }\end{array}$ & $\begin{array}{c}\text { Force de } \\
\text { cisaillement }\end{array}$ \\
\hline \multicolumn{8}{|c|}{ Abattage à même âge et poids différent } \\
\hline $\begin{array}{c}\text { Burson et al } \\
1980\end{array}$ & B & LT & 15 & $\begin{array}{l}0,8 \\
1,1 \\
1,4 \\
\end{array}$ & $\begin{array}{r}6,6 / 8 \\
+3,0 \% \text { (ns) } \\
+6,1 \% \text { (ns) } \\
\end{array}$ & & $\begin{array}{c}2,02 \mathrm{~kg} \\
-3,0 \%(\mathrm{~ns}) \\
-12,9 \% \text { (ns) }\end{array}$ \\
\hline $\begin{array}{c}\text { Roux et al } \\
1993\end{array}$ & V & $\mathrm{LT}$ & 60 & $\begin{array}{l}1,0 \\
1,1 \\
1,3 \\
\end{array}$ & & $\begin{array}{c}3,90 \% \\
+3,8 \%(\mathrm{~ns}) \\
+0,0 \%(\mathrm{~ns})\end{array}$ & $\begin{array}{c}1,41 \mathrm{~kg} \\
3,6 \%(\mathrm{~ns}) \\
8,0 \%(\mathrm{~ns})\end{array}$ \\
\hline $\begin{array}{c}\text { Gregory et al } \\
1994\end{array}$ & V & LT & 48 & & $\begin{array}{r}5,05 / 8 \\
+1,0 \% \text { (ns) } \\
\end{array}$ & $\begin{array}{r}3,78 \% \\
+9,5 \%\left({ }^{* \star}\right) \\
\end{array}$ & $\begin{array}{c}5,19 \mathrm{~kg} \\
-13,9 \% \text { (**) }^{*} \\
\end{array}$ \\
\hline $\begin{array}{c}\text { Muir et al } \\
2001\end{array}$ & B & LT & 24 & $\begin{array}{l}0,1 \\
0,7\end{array}$ & & & $\begin{array}{c}6,23 \mathrm{~kg} \\
-11,9 \%\left(^{*}\right)\end{array}$ \\
\hline \multirow{3}{*}{$\begin{array}{c}\text { Sinclair et al } \\
2001\end{array}$} & B & LL & 19 & $\begin{array}{l}0,9 \\
1,0 \\
\end{array}$ & $\begin{array}{r}15,3 / 24 \\
+5,2 \%(\mathrm{~ns}) \\
\end{array}$ & & $\begin{array}{c}5,29 \mathrm{~kg} \\
-8,3 \%(\mathrm{~ns}) \\
\end{array}$ \\
\hline & B & VL & 19 & $\begin{array}{l}0,9 \\
1,0 \\
\end{array}$ & $\begin{array}{r}13,5 / 24 \\
-3,7 \%(\mathrm{~ns}) \\
\end{array}$ & & \\
\hline & B & $\mathrm{BF}$ & 19 & $\begin{array}{l}0,9 \\
1,0 \\
\end{array}$ & $\begin{array}{r}10,4 / 24 \\
+3,8 \%(n s) \\
\end{array}$ & & \\
\hline \multirow{2}{*}{$\begin{array}{c}\text { Frickh et al } \\
2003\end{array}$} & G & $\mathrm{LT}$ & & & $\begin{array}{c}4,5 / 6 \\
+4,4 \% \text { (ns) } \\
\end{array}$ & $\begin{array}{c}4,8 \% \\
-27,1 \% \text { (ns) }\end{array}$ & $\begin{array}{c}3,3 \mathrm{~kg} \\
+9,1 \%(\mathrm{~ns}) \\
\end{array}$ \\
\hline & B & LT & & & $\begin{array}{c}4,0 / 6 \\
+5,0 \% \text { (ns) } \\
\end{array}$ & $\begin{array}{c}3,4 \% \\
-11,8 \%(n s) \\
\end{array}$ & $\begin{array}{c}2,8 \mathrm{~kg} \\
+25,0 \% \text { (ns) } \\
\end{array}$ \\
\hline $\begin{array}{c}\text { Cooke et al } \\
2004 \\
\end{array}$ & G & $\mathrm{LT}$ & & $\begin{array}{l}1,1 \\
1,4 \\
\end{array}$ & & $\begin{array}{c}1,5 \% \\
+40,4 \%(\mathrm{~ns}) \\
\end{array}$ & $\begin{array}{c}6,47 \mathrm{~kg} \\
-7,1 \%(\mathrm{~ns}) \\
\end{array}$ \\
\hline \multirow{2}{*}{$\begin{array}{l}\text { Sami et al } \\
\quad 2004\end{array}$} & $\mathrm{~T}$ & LT & 18 & $\begin{array}{l}1,0 \\
1,4 \\
\end{array}$ & $\begin{array}{c}3,82 / 6 \\
-12,8 \% \text { (ns) } \\
\end{array}$ & $\begin{array}{c}1,71 \% \\
+33,3 \%\left(^{\star}\right) \\
\end{array}$ & $\begin{array}{r}6,76 \mathrm{~kg} \\
+9,5 \% \text { (ns) } \\
\end{array}$ \\
\hline & $\mathrm{T}$ & LT & 18 & $\begin{array}{l}0,9 \\
1,4 \\
\end{array}$ & $\begin{array}{r}3,60 / 6 \\
+8,9 \% \text { (ns) } \\
\end{array}$ & $\begin{array}{c}1,58 \% \\
+74,7 \%\left(^{*}\right) \\
\end{array}$ & $\begin{aligned} & 5,91 \mathrm{~kg} \\
&+ 7,4 \% \text { (ns) } \\
&\end{aligned}$ \\
\hline \multirow{3}{*}{$\begin{array}{l}\text { Marino et al } \\
2006\end{array}$} & $\mathrm{~T}$ & $\mathrm{LT}$ & & $\begin{array}{l}0,9 \\
1,1\end{array}$ & $\begin{array}{r}7,06 / 10 \\
-7,9 \%(\mathrm{~ns}) \\
\end{array}$ & $\begin{array}{c}1,20 \% \\
+37,5 \%(\mathrm{~ns}) \\
\end{array}$ & $\begin{array}{c}1,48 \mathrm{~kg} \\
+51,4 \%(\mathrm{~ns}) \\
\end{array}$ \\
\hline & $\mathrm{T}$ & SM & & $\begin{array}{l}0,9 \\
1,1\end{array}$ & $\begin{array}{r}5,77 / 10 \\
+2,4 \%(n s)\end{array}$ & $\begin{array}{c}1,12 \% \\
+21,4 \% \\
\text { (ns) }\end{array}$ & $\begin{array}{c}1,93 \mathrm{~kg} \\
-1,0 \%(\mathrm{~ns})\end{array}$ \\
\hline & $\mathrm{T}$ & ST & & $\begin{array}{l}0,9 \\
1,1 \\
\end{array}$ & $\begin{array}{c}6,23 / 10 \\
-\quad 0,6 \%(n s) \\
\end{array}$ & $\begin{array}{c}0,78 \% \\
+30,8 \% \text { (ns) } \\
\end{array}$ & $\begin{array}{r}2,54 \mathrm{~kg} \\
+4,7 \% \text { (ns) } \\
\end{array}$ \\
\hline \multicolumn{8}{|c|}{ Abattage à même poids et âge différent } \\
\hline \multirow{3}{*}{$\begin{array}{c}\text { Dikeman et al } \\
1986\end{array}$} & $\mathrm{~B}$ & LT & & & & & $\begin{array}{c}5,94 \mathrm{~kg} \\
-9,1 \% \text { (ns) } \\
\end{array}$ \\
\hline & $\mathrm{T}$ & $\mathrm{LT}$ & & & & & $\begin{array}{r}6,71 \mathrm{~kg} \\
+14,9 \%\left(^{*}\right) \\
\end{array}$ \\
\hline & $B$ et $T$ & LT & & & $\begin{array}{c}7,17 / 10 \\
-0,4 \%(n s) \\
\end{array}$ & & \\
\hline $\begin{array}{c}\text { Mandell } \text { et } \text { al } \\
1998 \\
\end{array}$ & B & LT & & $\begin{array}{l}1,6 \\
1,7 \\
\end{array}$ & $\begin{array}{r}5,92 / 10 \\
+4,7 \%(\mathrm{~ns}) \\
\end{array}$ & $\begin{array}{c}4,6 \% \\
+10,9 \%(n s) \\
\end{array}$ & $\begin{array}{c}4,16 \mathrm{~kg} \\
-3,9 \% \text { (ns) } \\
\end{array}$ \\
\hline $\begin{array}{c}\text { Purchas et al } \\
2002 \\
\end{array}$ & $B$ et $T$ & $\mathrm{LT}$ & & $\begin{array}{l}0,4 \\
1,0 \\
\end{array}$ & & 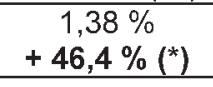 & $\begin{array}{c}10,89 \mathrm{~kg} \\
-19,7 \%\left(^{*}\right) \\
\end{array}$ \\
\hline $\begin{array}{c}\text { Cerdeno et al } \\
2006\end{array}$ & $\mathrm{~T}$ & LT & & $\begin{array}{l}1,2 \\
1,7 \\
\end{array}$ & $\begin{array}{c}6,0 / 10 \\
+0,0 \%(n s) \\
\end{array}$ & & $\begin{array}{c}5,7 \mathrm{~kg} \\
-15,8 \% \text { (ns) } \\
\end{array}$ \\
\hline
\end{tabular}

ns : écart non significatif ; $: p<0,05 ;{ }^{* *}: p<0,01 ; * * *: p<0,001$

$\mathrm{T}$ : taurillon, $\mathrm{B}$ : bœuf, G : génisse, V : vache ; LT: longissimus thoracis ; BF : biceps femoris ; LL : longissimus lumborum ; VL : vastus lateralis.

2004, Marino et al 2006) mais dans certains cas, elle peut diminuer de 12 à $14 \%$ (Gregory et al 1994, Muir et al 2001). L'augmentation du niveau alimentaire peut également conduire à une amélioration de la solubilité du collagène du muscle longissimus thoracis (Geay et al 2002, Sami et al 2004).
- Effet du niveau alimentaire lors d'un abattage à même poids

Chez les mâles entiers et castrés abattus à même poids, soit à 18 mois après un régime riche, soit à 26 mois après un régime modéré restreint, l'augmentation du niveau alimentaire de la ration conduit à une diminution significative dans le muscle longissimus thoracis à la fois de la dureté de la viande de 13,5\% et de la force de cisaillement d'environ $20 \%$ (Purchas et al 2002). Ces résultats sont probablement attribuables en partie à la différence d'âge à l'abattage entre les deux lots d'animaux. 
Chez des animaux jeunes, abattus à $9,12,15,18$ et 24 mois, l'augmentation du niveau alimentaire ne s'accompagne pas de modifications de la tendreté de la viande mais conduit à une augmentation de $15 \%$ de la force de cisaillement des viandes de taurillons sans modification de celle-ci dans le cas des viandes de bœufs (Dikeman et al 1986). Dans cet essai, les auteurs notent des valeurs de force de cisaillement anormalement élevées dans le cas des viandes de ces taurillons conduits à niveau alimentaire haut.

\section{- Effet du gain de poids}

Dans la mesure où les variations de niveau alimentaire sont généralement à l'origine de différence de gain de poids vif des animaux, les résultats concernant l'effet du niveau alimentaire sur la tendreté de la viande peuvent être analysés à la lumière des relations existant entre gain de poids des animaux et la tendreté de la viande.

L'augmentation du gain moyen quotidien de 600 à 1100 g/jour de 6893 animaux de différentes races (Angus, Hereford, Murray, Grey, Shorthorn, Brahman, Belmont Red et Santa Gertrudis) abattus à 220, 280 ou $340 \mathrm{~kg}$ de poids de carcasse semble susceptible de s'accompagner, selon un calcul de droites de régressions, d'une diminution des forces de cisaillement et de compression (Perry et Thompson 2005). Selon ces auteurs, cette plus faible résistance mécanique des muscles des animaux à croissance rapide pourrait s'expliquer soit par une augmentation de la protéolyse au cours de la maturation (Koohmaraie et al 2002) en lien avec une diminution de l'activité de la calpastatine (Shackelford et al 1994), soit par une diminution de la formation de liaisons stables entre les molécules de collagène (Harper 1999).

De la même façon, une étude sur la tendreté mesurée par évaluation sensorielle sur la viande de 100 génisses de race Charolaise a permis de conclure que l'augmentation du poids de carcasse de 360 à $380 \mathrm{~kg}$ d'animaux abattus à 33 mois s'accompagne d'une augmentation de $15 \%$ de la note de tendreté attribuée par un jury d'analyse sensorielle. Ainsi, indépendamment des pratiques d'élevage, il semble que l'augmentation du poids de carcasse des animaux pour un âge à l'abattage donné soit potentiellement favorable à la tendreté de la viande (Oury 2006). b) Influence de l'augmentation du niveau alimentaire en finition avec des aliments de nature différente

De nombreux essais évaluent l'effet de la nature des aliments distribués en finition sur la tendreté de la viande à niveau alimentaire identique ou différent. L'effet testé dans ce dernier cas n'est donc pas exclusivement celui de la nature de l'aliment en finition mais plutôt celui de l'interaction entre nature de l'aliment distribué et niveau alimentaire.

- Rations composées de fourrages de nature différente (tableau 5-A)

Rations à base d'ensilage d'herbe ou de foin

A mêmes apports nutritionnels ou à niveau alimentaire croissant, le passage d'une ration à base de foin à une ration à base d'ensilage d'herbe chez des bœufs ou des taurillons n'a pas d'effet sur la tendreté ou la force de cisaillement des muscles longissimus thoracis (Geay et al 1997, French et al 2000). Mais, le muscle semitendinosus des taurillons recevant du foin est en revanche significativement plus tendre d'environ $10 \%$ que celui des taurillons recevant en finition de l'ensilage d'herbe, en lien avec une moindre teneur et une plus forte solubilité du collagène (Geay et al 1997).

\section{Rations avec ou sans ensilage de $\underline{\text { maïs }}$}

Les rations à base d'ensilage de maïs en finition sont fréquemment comparées à celles composées de foin, d'herbe pâturée, d'aliments concentrés ou encore d'ensilage d'herbe, moins énergétiques. Par rapport au foin ou à l'herbe pâturée, elles peuvent conduire à des viandes dont la note de tendreté est de 9 à $22 \%$ plus élevée (Fishell et al 1985, Kogel et al 1998, Schwarz 2003), en lien, pour l'un des essais, avec une moindre force de cisaillement de 29,3\% (Kogel et al 1998). Néanmoins, cette évolution favorable de la tendreté n'est pas toujours vérifiée (Alberti et al 1992). Par rapport à l'ensilage d'herbe ou aux aliments concentrés, l'effet des rations à base d'ensilage de maïs est plus difficile à maître en évidence puisque les notes de tendreté et d'appréciation globale, les forces de cisaillement et les teneurs en lipides intramusculaires apparaissent équivalentes (Alberti et al 1992, Van Vooren et al 1992, Kogel et al 1998, Sinclair et al 1998, Frickh et al 2003).
- Rations de fourrages ou d'aliments concentrés (tableau 5-B)

$\underline{\text { Rations à base de foin ou d'ensilage }}$ de luzerne $v s$ rations à base d'aliments concentrés

L'augmentation du niveau alimentaire lorsque l'on passe d'une ration de foin ou d'ensilage de luzerne à une ration à base d'aliments concentrés (luzerne déshydratée ou maïs grain) n'améliore pas forcément la tendreté estimée par évaluation sensorielle ou par la force de cisaillement (Harrison et al 1978, Mandell et al 1998). A même niveau alimentaire, les rations à base de foin conduisent également à des viandes de même tendreté que les rations à base d'aliments concentrés (Cross et Dinius 1978, Alberti et al 1992). Ces résultats corroborent les conclusions rapportées par Geay et al (2002) qui indiquent que les notes de tendreté sont proches entre les viandes de bouvillons recevant des régimes à base de fourrages et celles de bouvillons recevant des régimes à base de céréales.

Rations d'herbe complémentées ou pas avec des aliments concentrés

Dans les quatre essais recensés réalisés sur bœufs et génisses, il n'existe pas de différence significative de tendreté et/ou de force de cisaillement entre les muscles longissimus thoracis des animaux finis avec de l'herbe pâturée complémentée ou pas d'aliments concentrés (Bowling et al 1978, Reagan et al 1981, French et al 2001, Baublits et al 2006). L'ajout d'aliments concentrés dans une ration d'herbe peut en revanche conduire à une augmentation des teneurs en lipides intramusculaires de 10 à $76 \%$ (Reagan et al 1981, French et al 2001, Baublits et al 2006).

Remplacement d'une ration d'herbe par une ration d'aliments concentrés

Il est difficile de conclure sur l'influence du remplacement d'une ration d'herbe par une ration d'aliments concentrés, dans la mesure où les conditions des différents essais diffèrent sensiblement.

Chez le taurillon Frison, une alimentation de 5 mois à l'auge est à l'origine de muscles longissimus thoracis dont la note de tendreté est $30 \%$ supérieure et dont la force de cisaillement est $25 \%$ inférieure en comparaison des animaux conduits à la pâture et abattus à même poids vif (Vestergaard et al 2000). Ces conclusions peuvent être attribuées à un effet positif de l'augmentation du niveau alimentaire des animaux conduit à l'auge. 
Tableau 5-A. Effet de la nature du fourrage distribué en finition sur la tendreté, la force de cisaillement et la teneur en lipides intramusculaires de la viande.

Les modifications sont exprimées en \% des valeurs obtenues pour le témoin. Le niveau de signification est indiqué entre parenthèses.

\begin{tabular}{|c|c|c|c|c|c|c|c|c|c|c|}
\hline Auteurs & $\begin{array}{l}\text { Ration } \\
\text { testée }\end{array}$ & $\begin{array}{l}\text { Type } \\
\text { d'animal }\end{array}$ & $\begin{array}{c}\text { Age } \\
\text { (mois) }\end{array}$ & $\begin{array}{c}\text { Gain de } \\
\text { poids en } \\
\text { finition ( }(\mathrm{j} / \mathrm{j})\end{array}$ & $\begin{array}{l}\text { Durée } \\
\text { de la } \\
\text { finition } \\
\text { (jour) }\end{array}$ & $\begin{array}{l}\text { Poids à } \\
\text { l'abattage }\end{array}$ & Muscle & $\begin{array}{l}\text { Note de } \\
\text { tendreté }\end{array}$ & $\begin{array}{c}\text { Force de } \\
\text { cisaillement }\end{array}$ & $\begin{array}{l}\text { Teneur en } \\
\text { Lipides } \\
\text { totaux }\end{array}$ \\
\hline \multicolumn{11}{|c|}{ Effet du remplacement de l'ensilage d'herbe par du foin } \\
\hline $\begin{array}{l}\text { Geay et al } \\
1997\end{array}$ & $\begin{array}{c}\text { EH } \\
\text { F } \\
\text { EH } \\
F\end{array}$ & $\begin{array}{l}\mathrm{T} \\
\mathrm{T}\end{array}$ & $\begin{array}{l}16 \\
16\end{array}$ & $\begin{array}{c}1093 \\
982 \\
1093 \\
982\end{array}$ & $\begin{array}{l}163 \\
163\end{array}$ & $\begin{array}{l}F<E H \\
F<E H\end{array}$ & $\begin{array}{l}\text { LT } \\
\text { ST }\end{array}$ & $\begin{array}{c}12,7 / 20 \\
-2,4 \% \text { (ns) } \\
9,6 / 20 \\
+9,4 \%\left(^{* *}\right)\end{array}$ & & \\
\hline $\begin{array}{l}\text { French et al } \\
2000\end{array}$ & $\begin{array}{c}\mathrm{EH} \\
\mathrm{F} \\
\end{array}$ & B & & & 85 & $\mathrm{~F}=\mathrm{EH}$ & LT & $\begin{array}{r}5,34 / 8 \\
+7,3 \% \text { (ns) } \\
\end{array}$ & $\begin{array}{c}3,63 \mathrm{~kg} \\
-6,5 \%(\mathrm{~ns}) \\
\end{array}$ & $\begin{array}{c}4,1 \% \\
-16,4 \%(n s) \\
\end{array}$ \\
\hline \multicolumn{11}{|c|}{ Effet du remplacement du fourrage ou des aliments concentrés de la ration par de l'ensilage de maïs } \\
\hline $\begin{array}{l}\text { Schwarz } \\
2003\end{array}$ & $\begin{array}{c}\mathrm{H} \\
\mathrm{EM}\end{array}$ & $\mathrm{G}$ & & $\begin{array}{c}652 \\
1071 \\
\end{array}$ & $\begin{array}{l}428 \\
277 \\
\end{array}$ & $\mathrm{H}=\mathrm{EM}$ & LT & $\begin{array}{c}4,0 / 6 \\
+12,5 \%\left(^{*}\right) \\
\end{array}$ & $\begin{array}{c}5,4 \mathrm{~kg} \\
-1,9 \% \text { (ns) } \\
\end{array}$ & \\
\hline $\begin{array}{l}\text { Fishell et al } \\
1985\end{array}$ & $\begin{array}{c}F \\
E M \\
F \\
E M\end{array}$ & $\begin{array}{l}B \\
B\end{array}$ & & $\begin{array}{c}770 \\
1420 \\
770 \\
1420\end{array}$ & $\begin{array}{l}120 \\
120 \\
120 \\
120\end{array}$ & $\begin{array}{l}F<E M \\
F<E M\end{array}$ & $\begin{array}{l}\text { LT } \\
\text { SM }\end{array}$ & $\begin{array}{c}5,64 / 8 \\
+9,8 \%\left(^{*}\right) \\
3,78 / 8 \\
+21,4 \%\left(^{*}\right)\end{array}$ & $\begin{array}{c}4,45 \mathrm{~kg} \\
-13,9 \% \text { (ns) } \\
4,48 \mathrm{~kg} \\
-7,6 \% \text { (ns) }\end{array}$ & \\
\hline $\begin{array}{l}\text { Alberti et al } \\
1992\end{array}$ & $\begin{array}{c}F \\
E M\end{array}$ & $\mathrm{~T}$ & & & & $\mathrm{~F}=\mathrm{EM}$ & LT & $\begin{array}{c}58,7 / 100 \\
-4,1 \% \text { (ns) }\end{array}$ & & \\
\hline $\begin{array}{l}\text { Kogel et al } \\
1998\end{array}$ & $\begin{array}{c}F \\
E M \\
E H \\
E M\end{array}$ & $\begin{array}{l}G \\
G\end{array}$ & $\begin{array}{l}18 \\
18\end{array}$ & & $\begin{array}{l}180 \\
180 \\
180 \\
180\end{array}$ & $\mathrm{EH}=\mathrm{EM}$ & LT & $\begin{array}{c}3,37 / 6 \\
+15,7 \%\left(^{*}\right) \\
3,65 / 6 \\
+6,8 \%(\mathrm{~ns})\end{array}$ & $\begin{array}{c}6,10 \mathrm{~kg} \\
-11,8 \% \text { (ns) } \\
5,34 \mathrm{~kg} \\
+0,7 \% \text { (ns) }\end{array}$ & $\begin{array}{c}1,67 \% \\
+29,3 \%\left(^{*}\right) \\
2,05 \% \\
+5,4 \%(n s)\end{array}$ \\
\hline $\begin{array}{l}\text { Frickh et al } \\
2003\end{array}$ & $\begin{array}{l}\text { EH } \\
\text { EM } \\
\text { EH } \\
\text { EM }\end{array}$ & $\begin{array}{l}\mathrm{G} \\
\mathrm{B}\end{array}$ & & & & $\begin{array}{l}E H=E M \\
E H=E M\end{array}$ & $\begin{array}{l}\text { LT } \\
\text { LT }\end{array}$ & $\begin{array}{c}4,3 / 6 \\
+7,0 \% \text { (ns) } \\
4,2 / 6 \\
-9,3 \% \text { (ns) } \\
\end{array}$ & $\begin{array}{c}3,5 \mathrm{~kg} \\
-5,7 \%(\mathrm{~ns}) \\
3,6 \mathrm{~kg} \\
-11,1 \%(\mathrm{~ns}) \\
\end{array}$ & $\begin{array}{c}3,5 \% \\
+28,6 \% \text { (ns) } \\
3,0 \% \\
+13,3 \% \text { (ns) } \\
\end{array}$ \\
\hline $\begin{array}{l}\text { Juniper et al } \\
2005\end{array}$ & $\begin{array}{l}\mathrm{EH} \\
\mathrm{EM}\end{array}$ & B & & $\begin{array}{c}1262 \\
920\end{array}$ & $\begin{array}{l}160 \\
160\end{array}$ & $\mathrm{EH}<\mathrm{EM}$ & LT & $\begin{array}{c}50,9 / 100 \\
-0,8 \% \text { (ns) }\end{array}$ & & \\
\hline $\begin{array}{l}\text { Alberti et al } \\
1992\end{array}$ & $\begin{array}{c}C \\
\mathrm{EM}\end{array}$ & T & & $\begin{array}{l}1300 \\
1400\end{array}$ & & $\mathrm{C}=\mathrm{EM}$ & LT & $\begin{array}{c}55,8 / 100 \\
+0,9 \%(\mathrm{~ns})\end{array}$ & & \\
\hline $\begin{array}{l}\text { Van Vooren } \\
\text { et al } 1992\end{array}$ & $\begin{array}{c}\mathrm{C} \\
\mathrm{EM}\end{array}$ & $T$ & & $\begin{array}{l}1300 \\
1400\end{array}$ & & $\mathrm{EM}<\mathrm{C}$ & LT & & $\begin{array}{c}4,25 \mathrm{~kg} \\
-2,9 \% \text { (ns) }\end{array}$ & $\begin{array}{c}0,6 \% \\
+16,7 \% \text { (ns) } \\
\end{array}$ \\
\hline $\begin{array}{l}\text { Sinclair et al } \\
1998\end{array}$ & $\begin{array}{c}C \\
\text { EM } \\
C \\
E M \\
C \\
E M\end{array}$ & $\mathrm{~T}$ & $\begin{array}{r}13,5 \\
13,5 \\
13,5\end{array}$ & $\begin{array}{l}1120 \\
1550 \\
1120 \\
1550 \\
1120 \\
1550 \\
\end{array}$ & $\begin{array}{l}147 \\
147 \\
147 \\
147 \\
147 \\
147 \\
\end{array}$ & $\begin{array}{l}\mathrm{EM}<\mathrm{C} \\
\mathrm{EM}<\mathrm{C}\end{array}$ & $\begin{array}{l}\text { BF } \\
\text { SM }\end{array}$ & $\begin{array}{c}5,2 / 8 \\
+1,9 \%(\mathrm{~ns}) \\
3,8 / 8 \\
+0,0 \%(\mathrm{~ns}) \\
4,3 / 8 \\
+0,0 \%(\mathrm{~ns})\end{array}$ & & \\
\hline
\end{tabular}

EM : ensilage de maïs ; H : herbe ; C : aliments concentrés ; F : foin ; EH : ensilage d'herbe ; T : taurillon, $B$ : bœuf, G : génisse, V : vache. LT : longissimus thoracis ; ST : semitendinosus ; SM : semimembranosus ; BF : biceps femoris ; LL : longissimus lumborum.

${ }^{*}: p<0,05 ;{ }^{* *}: p<0,01$.

Chez les bœufs abattus à même poids de carcasse, la finition à base d'aliments concentrés conduit à des muscles biceps femoris dont la note de tendreté est $9 \%$ plus faible et dont la force de cisaillement est $22 \%$ plus élevée en comparaison d'une finition à base d'herbe (Wu et al 1981). Ces résultats peuvent en partie être attribués à l'effet positif de l'activité physique sur la tendreté des muscles locomoteurs. Ainsi, dans ce travail, aucun effet significatif n'a été mis en évidence sur le muscle longissimus thoracis. Realini et al (2004) ne mettent pas en évidence d'écart significatif de force de cisaillement des viandes après un jour de maturation entre une conduite à l'auge avec des aliments concentrés et une conduite à la pâture. Néanmoins, la conduite à la pâture semble être plus favorable à la maturation des viandes dans la mesure où, après 7 à 14 jours de maturation, les animaux alimentés avec des aliments concentrés conduisent à des viandes dont la force de cisaillement est $7 \%$ plus élevée que celle des animaux conduits à la pâture (Realini et al 2004).

\section{3 / Effet de l'allongement de la durée de finition}

L'étude de la durée de finition est envisagée de deux manières différentes selon que les animaux sont abattus à poids égal (tableau 6-A) ou différent (tableau 6-B). Les essais répertoriés portent généralement sur des comparaisons de durées de finition à même niveau énergétique. Cependant, certains auteurs testent la durée de finition à niveau énergétique différent.

Lorsque les animaux sont abattus à même poids de carcasse, il n'y a pas dans la plupart des essais de différence des propriétés sensorielles ou rhéologiques des viandes selon la durée de finition malgré une augmentation de la teneur en lipides intramusculaires des viandes (Dinius et Cross 1978, Tatum et al 1980, Crouse et al 1986, Camfield et al 1997). Dans certains cas, l'augmentation de la durée de finition peut conduire à une diminution de plus de $26 \%$ de la force de cisaillement des viandes de boufs finis en 147 ou 175 jours (Burson et al 1980). Ce dernier résultat va dans le même sens que 
Tableau 5-B. Effet de la distribution d'aliments concentrés en finition à la place ou en complément des fourrages sur la tendreté, la force de cisaillement et la teneur en lipides intramusculaires de la viande.

Les modifications sont exprimées en \% des valeurs obtenues pour le témoin.

Le niveau de signification est indiqué entre parenthèses.

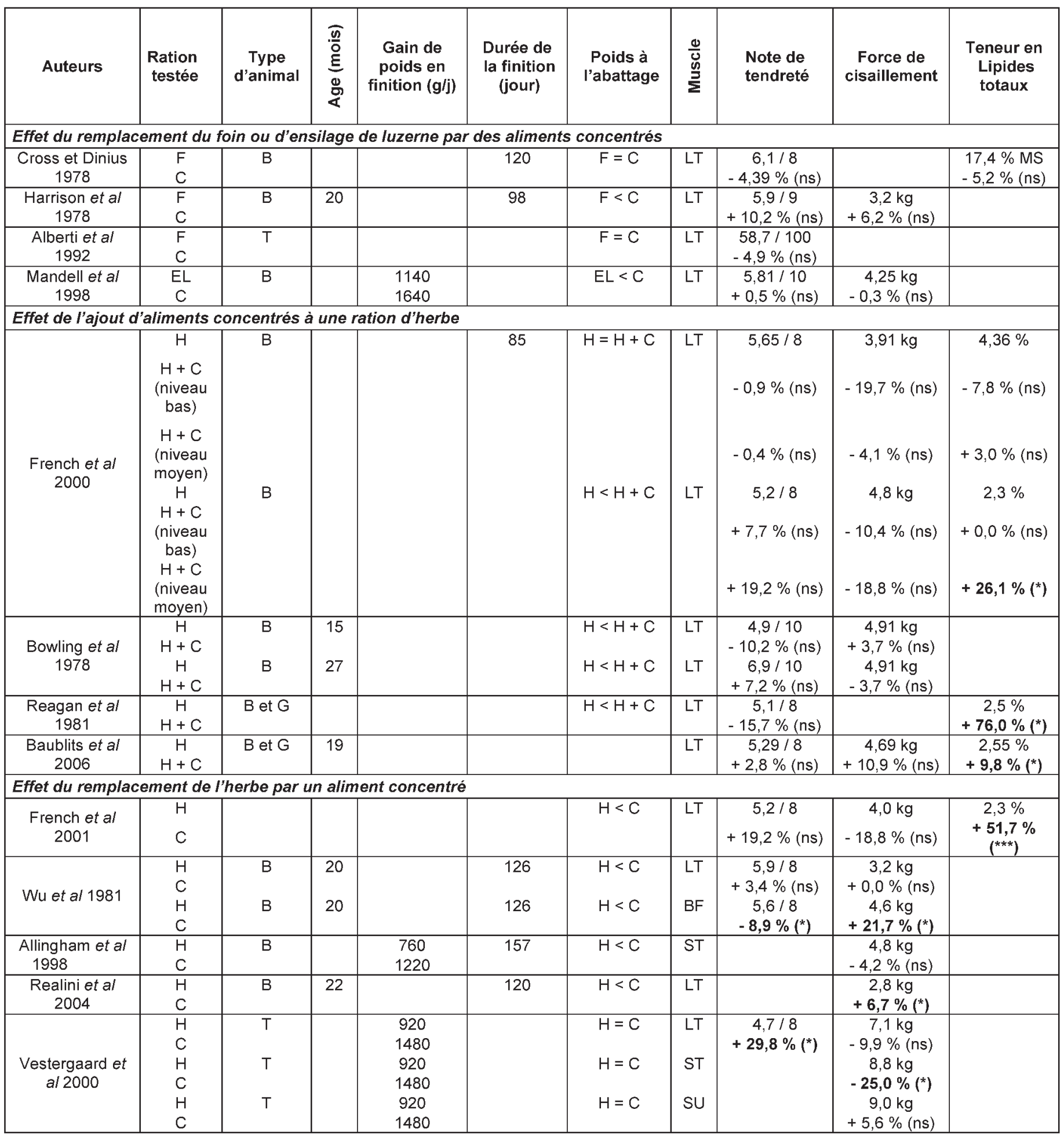

$\mathrm{H}$ : herbe ; $\mathrm{C}$ : aliments concentrés ; $\mathrm{F}$ : foin ; $\mathrm{EL}$ : ensilage de luzerne ; $\mathrm{T}$ : taurillon, $\mathrm{B}$ : bœuf, $\mathrm{G}$ : génisse ;

LT : longissimus thoracis ; T : semitendinosus ; BF : biceps femoris ; SU : supraspinatus ; ns : pas de modification significative ; ${ }^{*}$ : $<0,05$.

l'évolution des teneurs et solubilités du collagène au cours de la finition pourrait expliquer cette amélioration. Ainsi, entre $0,14,28,46$ et 56 jours de finition, la teneur en collagène total du muscle biceps femoris de vaches adul- tes diminue tandis qu'en parallèle sa solubilité augmente (Schnell et al 1997). Ces effets sur la solubilité du collagène se retrouvent entre $0,28,56$ et 84 jours de finition (Boleman et al 1996).
Lorsque les animaux qui ont eu une période de finition plus longue sont abattus plus lourds, le jury d'analyse sensorielle n'est pas en mesure, dans la majorité des études (8/11) de différencier de façon significative les viandes 
Tableau 6-A. Effet de la durée de la finition des animaux sur la tendreté, la force de cisaillement et la teneur en lipides intramusculaires des muscles longissimus thoracis, Comparaison pour un abattage à même poids de carcasse.

Les modifications sont exprimées en \% des valeurs obtenues pour le témoin (durée de finition la plus courte). Le niveau de signification est indiqué entre parenthèses.

\begin{tabular}{|c|c|c|c|c|c|c|c|}
\hline Auteurs & $\begin{array}{l}\text { Durée de } \\
\text { finition } \\
\text { (jours) }\end{array}$ & $\begin{array}{l}\text { Poids de } \\
\text { carcasse } \\
(\mathrm{kg})\end{array}$ & $\begin{array}{l}\text { Type de } \\
\text { conduite }\end{array}$ & $\begin{array}{c}\text { Type } \\
\text { d'animal }\end{array}$ & $\begin{array}{l}\text { Teneur en } \\
\text { lipides } \\
\text { totaux }\end{array}$ & $\begin{array}{l}\text { Note de } \\
\text { tendreté }\end{array}$ & $\begin{array}{l}\text { Force de } \\
\text { cisaillement }\end{array}$ \\
\hline \multirow{4}{*}{$\begin{array}{c}\text { Dinius et Cross } \\
1978\end{array}$} & 0 & 251 & & \multirow[t]{3}{*}{ Bœuf } & $12,5 \% \mathrm{MS}$ & \multirow{4}{*}{$\begin{array}{c}3,9 / 8 \\
+15,4 \% \\
\text { (ns) } \\
+17,9 \% \\
\text { (ns) } \\
4,6 / 8 \\
-2,2 \% \text { (ns) }\end{array}$} & \multirow{2}{*}{$\begin{array}{c}8,5 \mathrm{~kg} \\
-10,6 \% \text { (ns) }\end{array}$} \\
\hline & 21 & 250 & & & $-10,4 \%(n s)$ & & \\
\hline & 42 & 267 & & & $+26,4 \%\left(^{*}\right)$ & & $-20,2 \%$ (ns) \\
\hline & $\begin{array}{l}42 \\
63\end{array}$ & $\begin{array}{l}267 \\
290\end{array}$ & & Bœuf & $\begin{array}{r}15,8 \% \mathrm{MS} \\
+23,4 \%\left(^{*}\right)\end{array}$ & & $\begin{array}{r}6,8 \mathrm{~kg} \\
+44 \% \text { (ns) }\end{array}$ \\
\hline \multirow{6}{*}{$\begin{array}{l}\text { Burson et al } \\
1980\end{array}$} & 56 & 176 & Niveau bas & \multirow[t]{2}{*}{ Bœuf } & & $\begin{array}{c}-2,2 \% \text { (ns) } \\
6,6 / 8\end{array}$ & $2,02 \mathrm{~kg}$ \\
\hline & 91 & 190 & & & & $+3,0 \%$ (ns) & $-0,5 \%$ (ns) \\
\hline & 91 & 190 & Niveau bas & Bœuf & & $6,8 / 8$ & $2,01 \mathrm{~kg}$ \\
\hline & 119 & 207 & & & & $+0,0 \%(n s)$ & $+7,0 \%(\mathrm{~ns})$ \\
\hline & 147 & 293 & Niveau haut & Bœuf & & $6,6 / 8$ & $2,35 \mathrm{~kg}$ \\
\hline & 175 & 297 & & & & $+7,6 \%$ (ns) & $-26,8 \%\left(^{*}\right)$ \\
\hline \multirow{3}{*}{$\begin{array}{c}\text { Tatum et al } \\
1980\end{array}$} & 100 & 321 & & \multirow[t]{3}{*}{ Bœuf } & & $5,75 / 8$ & $4,39 \mathrm{~kg}$ \\
\hline & 130 & 319 & & & & $-0,7 \%$ (ns) & $-1,8 \%$ (ns) \\
\hline & 160 & 328 & & & & $+1,4 \%(n s)$ & $-3,0 \%$ (ns) \\
\hline \multirow{2}{*}{$\begin{array}{c}\text { Crouse et al } \\
1986\end{array}$} & 30 & 118 & & \multirow[t]{2}{*}{ Taurillons } & & $5,18 / 8$ & $5,9 \mathrm{~kg}$ \\
\hline & 60 & 118 & & & & $-4,2 \%(n s)$ & $+5,1 \%(n s)$ \\
\hline \multirow{4}{*}{$\begin{array}{c}\text { Camfield et al } \\
1997\end{array}$} & 0 & 244 & & \multirow{4}{*}{$\begin{array}{l}\text { Bœuf } \\
\text { Bœuf }\end{array}$} & & \multirow{4}{*}{$\begin{array}{c}4,28 / 8 \\
+5,4 \% \text { (ns) } \\
4,33 / 8 \\
+11,8 \% \\
\text { (ns) }\end{array}$} & \multirow{4}{*}{$\begin{array}{c}4,59 \mathrm{~kg} \\
-11,5 \% \text { (ns) } \\
4,05 \mathrm{~kg} \\
-2,0 \% \text { (ns) }\end{array}$} \\
\hline & 30 & 241 & & & & & \\
\hline & 60 & 266 & & & & & \\
\hline & 90 & 275 & & & & & \\
\hline
\end{tabular}

ns : résultat non significatif ; * $: p<0,05$.

issues d'animaux ayant eu une finition durant entre 0 et 63 jours (Dinius et Cross 1978), 0 et 84 jours (Boleman et al 1996), 0 et 90 jours (Camfield et al 1997), 56 et 175 jours (Burson et al 1980) ou encore 90 et 170 jours (Xie et al 1996) (tableau 6-B).

De même, dans 7 cas sur 10 il n'apparaît pas de différence de force de cisaillement entre les viandes selon que les durées de finition sont entre 0 et 63 jours (Dinius et Cross 1978), 0 et 90 jours (Camfield et al 1997), 40 et 98 jours (Harrison et al 1978), 100 et 138 jours (Sami et al 2004) ou entre 105 et 147 jours (Van Koevering et al 1995).

Dans certains cas cependant, l'augmentation de la durée de finition, en conduisant à une augmentation parallèle du poids de carcasse des animaux, s'accompagne d'une amélioration de la tendreté des viandes. Ainsi, abattus plus lourds, les animaux engraissés pendant la période la plus longue ont, dans certains essais, des viandes dont la note de tendreté est entre 17 et $45 \%$ plus élevée (Harrison et al 1978, Schnell et al 1997, Sami et al 2004). De même, l'augmentation de la durée de finition semble s'accompagner dans de nombreux cas d'une augmentation de 21 à $75 \%$ des teneurs en lipides intramusculaires (Dinius et Cross 1978, Van Koevering et al 1995, Sami et al 2004). L'amélioration de la solubilité du collagène des muscles lon- gissimus thoracis avec l'allongement de la durée de finition semble pouvoir, comme précédemment expliquer en partie l'amélioration de la tendreté de la viande (Sami et al 2004). Cependant, ponctuellement, certains auteurs peuvent mettre en évidence une augmentation de la force de cisaillement avec l'allongement de la durée de finition (Xie et al 1996) sans que cela se répercute significativement sur les notes de tendreté évaluées par le jury. Cette augmentation de la résistance mécanique pourrait être attribuée pour partie à l'augmentation d'environ 3 mois de l'âge à l'abattage des bœufs.

\section{Conclusion}

Dans cette revue bibliographique, les effets significatifs de plusieurs facteurs d'élevage ont pu être mis en évidence à partir des résultats expérimentaux retranscrits dans 86 articles scientifiques.

Sur les 86 références bibliographiques compilées, 62 ne révèlent pas d'effet significatif d'un facteur déterminé sur la tendreté ou la force de cisaillement ou de compression des viandes. On sait que la variabilité des notes de tendreté globale du muscle longissimus thoracis obtenue par un jury est généralement comprise entre
$15,0 \%$ et $27 \%$ selon la taille de l'échantillon analysé (30 à 100 animaux) (Renand et al 1997, 2001, Maher et al 2004, Rhee et al 2004). La faible part de résultats significatifs peut en partie être attribuée à ce niveau de variabilité non négligeable des résultats sensoriels. Il est également vraisemblable que la variabilité individuelle des animaux ait limité les différences significatives entre lots, d'autant plus qu'au sein des essais, les animaux peuvent être abattus à âges, à poids ou bien à état d'engraissement différents. Enfin, les différents travaux reportés dans cette étude bibliographique concernent des races et des muscles distincts et il est probable que les effets des facteurs d'élevage varient selon ces deux critères.

Globalement, il apparaît que la tendreté et la force de cisaillement de la viande chez le bovin mâle n'évoluent pas avant l'âge de 2 ans ( 0 cas sur 10$)$, celles de la viande de femelles sont stables jusqu'à 35 mois (dans 4 cas sur 4 ) et peuvent dans certains cas évoluer négativement au-delà de cet âge (dans 4 essais sur 5). Chez la femelle, la gestation et le vêlage ne détériorent pas la tendreté de la viande, que les génisses soient abattues à même âge (4 essais sur 4) ou plus jeunes (3 essais sur 4) que les primipares. Un auteur a néanmoins mis en évidence une interaction négative entre les facteurs âge à l'abat- 
Tableau 6-B. Effet de la durée de finition des animaux sur la tendreté, la force de cisaillement et la teneur en lipides intramusculaires des muscles longissimus thoracis. Comparaison pour un abattage à poids de carcasse croissant.

Les modifications sont exprimées en \% des valeurs obtenues pour le témoin (durée de finition la plus courte). Le niveau de signification est indiqué entre parenthèses.

\begin{tabular}{|c|c|c|c|c|c|c|c|}
\hline Auteurs & $\begin{array}{c}\text { Durées } \\
\text { de } \\
\text { finition } \\
\text { (jours) } \\
\end{array}$ & $\begin{array}{l}\text { Poids de } \\
\text { carcasse } \\
\text { (kg) }\end{array}$ & $\begin{array}{l}\text { Type de } \\
\text { conduite }\end{array}$ & $\begin{array}{c}\text { Type } \\
\text { d'animal }\end{array}$ & $\begin{array}{l}\text { Teneur en } \\
\text { lipides } \\
\text { totaux }\end{array}$ & $\begin{array}{l}\text { Note de } \\
\text { tendreté }\end{array}$ & $\begin{array}{l}\text { Force de } \\
\text { cisaillement }\end{array}$ \\
\hline \multirow{2}{*}{$\begin{array}{c}\text { Dinius et Cross } \\
1978\end{array}$} & 0 & 251 & & Bœuf & $12,5 \% \mathrm{MS}$ & $3,9 / 8$ & $8,5 \mathrm{~kg}$ \\
\hline & $\begin{array}{l}63 \\
21 \\
63\end{array}$ & $\begin{array}{l}250 \\
251 \\
267\end{array}$ & & Bœuf & $\begin{array}{c}+56,0 \%\left(^{*}\right) \\
11,2 \% \mathrm{MS} \\
+74,1 \%\left(^{*}\right)\end{array}$ & $\begin{array}{c}+15,4 \%(\mathrm{~ns}) \\
4,5 / 8 \\
+0,0 \%(\mathrm{~ns}) \\
\end{array}$ & $\begin{array}{c}-16,5 \% \text { (ns) } \\
7,6 \mathrm{~kg} \\
-6,6 \% \text { (ns) }\end{array}$ \\
\hline $\begin{array}{c}\text { Harrison et al } \\
1978\end{array}$ & $\begin{array}{l}40 \\
98\end{array}$ & $\begin{array}{l}279 \\
324\end{array}$ & & Bœuf & & $\begin{array}{c}5,3 / 9 \\
+22,6 \%\left(^{*}\right)\end{array}$ & $\begin{array}{c}3,2 \mathrm{~kg} \\
6,2 \%(\mathrm{~ns})\end{array}$ \\
\hline $\begin{array}{l}\text { Van Koevering } \\
\text { et al } 1995\end{array}$ & $\begin{array}{l}105 \\
119 \\
119 \\
133 \\
133 \\
147 \\
\end{array}$ & $\begin{array}{l}308 \\
325 \\
325 \\
335 \\
335 \\
347 \\
\end{array}$ & & $\begin{array}{l}\text { Bœuf } \\
\text { Bœuf } \\
\text { Bœuf }\end{array}$ & $\begin{array}{c}3,01 \% \\
+21,6 \% \text { (*) }^{*} \\
3,66 \% \\
+2,5 \%(\mathrm{~ns}) \\
3,75 \% \\
+6,7 \%(\mathrm{~ns}) \\
\end{array}$ & & $\begin{array}{c}4,45 \mathrm{~kg} \\
-0,7 \% \text { (ns) } \\
4,42 \mathrm{~kg} \\
-7,0 \% \text { (ns) } \\
4,11 \mathrm{~kg} \\
-1,2 \%(\mathrm{~ns})\end{array}$ \\
\hline $\begin{array}{c}\text { Boleman et al } \\
1996\end{array}$ & $\begin{array}{c}0 \\
28 \\
28 \\
56 \\
56 \\
84\end{array}$ & $\begin{array}{l}284 \\
301 \\
301 \\
333 \\
333 \\
362 \\
\end{array}$ & & $\begin{array}{l}\text { Vache } \\
\text { Vache } \\
\text { Vache }\end{array}$ & & $\begin{array}{c}4,7 / 8 \\
+0,0 \% \text { (ns) } \\
4,7 / 8 \\
+2,1 \% \text { (ns) } \\
4,8 / 8 \\
+2,1 \% \text { (ns) }\end{array}$ & $\begin{array}{c}7,1 \mathrm{~kg} \\
-5,6 \% \text { (ns) } \\
6,7 \mathrm{~kg} \\
-14,9 \% \text { (ns) } \\
5,7 \mathrm{~kg} \\
-12,3 \% \text { (ns) }\end{array}$ \\
\hline $\begin{array}{c}\text { Xie et al } \\
1996\end{array}$ & $\begin{array}{c}90 \\
170\end{array}$ & $\begin{array}{l}381 \\
448\end{array}$ & & Bœuf & & $\begin{array}{c}5,33 / 8 \\
-15,4 \% \text { (ns) } \\
\end{array}$ & $\begin{aligned} & 2,78 \mathrm{~kg} \\
&+ 27,0 \%\left(^{\star}\right) \\
&\end{aligned}$ \\
\hline $\begin{array}{c}\text { Camfield et al } \\
1997\end{array}$ & $\begin{array}{l}0 \\
60 \\
90 \\
30 \\
60 \\
90 \\
\end{array}$ & $\begin{array}{l}244 \\
266 \\
275 \\
241 \\
266 \\
275 \\
\end{array}$ & & Bœuf & & $\begin{array}{c}4,28 / 8 \\
+1,2 \% \text { (ns) } \\
+13,1 \% \text { (ns) } \\
4,51 / 8 \\
-4,0 \%(n s) \\
+7,3 \%(n s)\end{array}$ & $\begin{array}{c}4,59 \mathrm{~kg} \\
-11,8 \% \text { (ns) } \\
-13,5 \% \text { (ns) } \\
4,06 \mathrm{~kg} \\
-0,2 \% \text { (ns) } \\
-2,2 \% \text { (ns) } \\
\end{array}$ \\
\hline $\begin{array}{c}\text { Schnell et al } \\
1997\end{array}$ & $\begin{array}{c}0 \\
28 \\
42 \\
56 \\
14 \\
28 \\
42 \\
56\end{array}$ & $\begin{array}{l}237 \\
295 \\
298 \\
319 \\
256 \\
295 \\
298 \\
319\end{array}$ & & Vache & & $\begin{array}{c}4,75 / 8 \\
+8,6 \%(\mathrm{~ns}) \\
-6,5 \%(\mathrm{~ns}) \\
+18,1 \%\left(^{*}\right) \\
+3,87 / 8 \\
+33,3 \%\left(^{*}\right) \\
+14,7 \%(\mathrm{~ns}) \\
+45,0 \%\left(^{*}\right)\end{array}$ & $\begin{array}{c}3,94 \mathrm{~kg} \\
+9,9 \%(\mathrm{~ns}) \\
-2,3 \%(\mathrm{~ns}) \\
+3,3 \%(\mathrm{~ns}) \\
5,17 \mathrm{~kg} \\
-6,2 \%(\mathrm{~ns}) \\
-25,5 \%(\mathrm{~ns}) \\
-21,3 \%(\mathrm{~ns})\end{array}$ \\
\hline $\begin{array}{l}\text { Sami et al } \\
\quad 2004\end{array}$ & $\begin{array}{l}100 \\
138 \\
100 \\
138 \\
\end{array}$ & $\begin{array}{l}358 \\
385 \\
324 \\
351 \\
\end{array}$ & $\begin{array}{l}\text { Intensive } \\
\text { Extensive }\end{array}$ & Bœuf & $\begin{array}{c}2,28 \% \mathrm{MS} \\
\left.+21,1 \% \text { ( }^{*}\right) \\
1,71 \% \mathrm{MS} \\
-7,6 \%(\mathrm{~ns}) \\
\end{array}$ & $\begin{array}{c}3,33 / 6 \\
+17,7 \%\left(^{*}\right) \\
3,82 / 6 \\
-5,8 \%(n s) \\
\end{array}$ & $\begin{array}{c}7,40 \mathrm{~kg} \\
-14,2 \% \text { (ns) } \\
6,76 \mathrm{~kg} \\
-12,6 \% \text { (ns) }\end{array}$ \\
\hline
\end{tabular}

ns : résultat non significatif ; * $: p<0,05$.

tage et vêlage en défaveur de la viande de primipares, moins tendre que celle des nullipares. L'effet de l'activité physique semble être favorable à la tendreté des muscles locomoteurs $(2$ cas sur 2) mais n'influence pas celle des muscles non locomoteurs (2 cas sur 2). L'ensemble des 8 auteurs recensés s'accorde pour dire que, intra-race, le type morphologique n'a pas impact significatif sur la tendreté des viandes et la force de cisaillement des muscles.

Concernant les facteurs alimentaires, il ressort que l'augmentation du niveau alimentaire n'influence généralement pas la tendreté de la viande ou la force de cisaillement du muscle de façon significative (dans 8 cas sur 13). Les effets bénéfiques de la croissance compensatrice, évalués uniquement sur les animaux jeunes (taurillons, bœufs et génisses) doivent en revanche être confirmés, dans la mesure où les résultats apparaissent variables selon les conditions des expérimentations. L'allongement de la durée de finition n'a, dans un certain nombre d'essais, pas d'effet significatif sur la tendreté et la force de cisaillement (dans 7 cas sur 13). Néanmoins, certains essais ont pu conclure à un effet positif de l'allongement de la durée de finition (dans 5 essais sur 13). Cette étude bibliographique montre enfin qu'un certain nombre d'imprécisions demeurent dans l'évaluation de l'effet de la nature de l'alimentation sur la tendreté. Les niveaux alimentaires des rations testées peuvent en effet être équivalents ou très différents d'une expérimentation à l'autre. De même, les travaux peuvent concerner des animaux de catégorie, d'âge et poids très variables ce qui engendre des difficultés dans la mise en évidence de l'effet de l'alimentation sur la tendreté.

Ces différents travaux prennent plus ou moins en compte les interactions entre les différents facteurs. Or, en élevage, les différents facteurs de conduite sont peu dissociables les uns des autres. Il serait donc tout à fait opportun de développer des approches intégratives sur un nombre conséquent d'animaux, prenant en considération non plus l'effet individuel de facteurs d'élevage mais leurs associations, afin d'évaluer leurs répercutions sur les performances des animaux à l'abattage et la qualité sensorielle des viandes bovines. 


\section{Références}

Aalhus J.L., Price M.A., Shand P.J., Hawrysh Z., 1991. Endurance-exercised growing-sheep: tenderness increase and change in meat quality. Meat Sci., 29, 57-68.

Alberti P., Sanudo C., Santolaria P., Touraille C., 1992. Sensory evaluation of meat in young bulls fed with different diets. $38^{\text {th }}$ Int. Cong. Meat Sci. Technol., Clermont-Ferrand, France, 221-224.

Allingham P.G., Harper G.S., Hunter R.A., 1998. Effect of growth path on the tenderness of the semitendinosus muscle of Brahman-cross steers. Meat Sci., 48, 65-73.

Altarriba J., Varona L., Moreno C., Yagüe G., Sanudo C., 2005. Consequences of selection for growth on carcass and meat quality in Pirenaica cattle. Liv. Prod. Sci., 95, 103-114.

Bastien D., Denoyelle C., Tribot Laspiere P., 2002. Age à l'abattage, suspension pelvienne, pratique de l'affranchi : pour une meilleure argumentation des choix techniques en matière de gestion de la tendreté dans les démarches qualité. Renc. Rech. Rum., 9, 251-254.

Baublits R.T., Brown Jr A.H., Pohlman F.W., Rule D.C., Johnson Z.B., Onks D.O., Murrieta C.M., Rochards C.J., Loveda H.D., Sandeln B.A., Pugh R B., 2006. Fatty acid and sensory characteristics of beef from three biological types of cattle grazing cool-season forages supplemented with soyhulls. Meat Sci., 72, 100-107.

Berge P., Damergi C., Culioli J., Boccard R., 1991. Effets des conditions d'élevage sur la qualité de la viande bovine : influence du mode de stabulation et d'un brossage dorsal. Viandes et Produits Carnés, 12, 167-170.

Bindon B.M., Jones N.M., 2001. Cattle supply, production systems and market for Australian beef. Aust. J. Exp. Agric., 41, 861877.

Boleman S.J., Miller R.K., Buyck M.J., Cross H.R., Savell J.W., 1996. Influence of re-alimentation of mature cows on maturity, color, collagen solubility, and sensory characteristics. J. Anim. Sci., 74, 2187-2194.

Boleman S.J., Boleman S.L., Miller R.K., Taylor J.F., Cross H.R., Wheeler T.L., Koohmaraie M., Shackelford S.D., Miller M.F., West R.L., Johnson D.D., Savell J.W., 1997. Consumer evaluation of beef of known categories of tenderness. J. Anim. Sci., 75, 15211524.

Bowling R.A., Riggs J.K., Smith G.C., Carpenter Z.L., Reddish R.L., Butler O.D., 1978. Production, carcass and palatability characteristics of steers produced by different management systems. J. Anim. Sci., 46, 333-340.

Burson D.E., Hunt M.C., Allen D.M., Kastner C.L., Kropf D.H., 1980. Diet energy density and time on feed effects on beef longissimus muscle palatability. J. Anim. Sci., 51, 875-881.

Cabaraux J.F., Hornick J.L., Dotreppe O., Dufrasne I., Clinquart A., Istasse L., 2004. Effects of the calving number on animal performance, carcass and meat composition in finishing Belgian Blue double-muscled culled females. Liv. Prod. Sci., 87, 161-169.

Camfield P.K., Brown A.H., Lewis P.K., Rakes L.Y., Johnson Z.B., 1997. Effects of frame size and time-on-feed on carcass characteristics, sensory attributes, and fatty acid profiles of steers. J. Anim. Sci., 75, 1837-1844.
Cepin S., Cepon M., Skorjanc D., 1992. Influence of growth and carcass weight on carcass and meat characteristics of Brown Bulls. $38^{\text {th }}$ Int. Cong. Meat Sci. Technol., ClermontFerrand, France, 9-12.

Cerdeno A., Vieira C., Serrano E., Lavin P., Mantecon A.R., 2006. Effects of feeding strategy during a short finishing period on performance, carcass and meat quality in previously-grazed young bulls. Meat Sci., 72, 719-726.

Combris P., 2003. Cinquante ans de consommation de viande et de produits laitiers en France. Renc. Rech. Rum., 10, 327-330.

Cooke D.W.I., Monahan F.J., Brophy P.O., Boland M.P., 2004. Comparison of concentrates or concentrates plus forages in a total mixed ration or discrete ingredient format: effects on beef production parameters and on beef composition, colour, texture and fatty acid profile. Ir. J. Agric. Fd Res., 43, 201-216.

Cross H.R., Dinius D.A., 1978. Carcass and palatability characteristics of beef steers finished on forage diets. J. Anim. Sci., 47, 1265-1271.

Crouse J.D., Calkins C.R., Seideman S.C., 1986. The effects of rate of change in body weight on tissue development and meat quality of youthful bulls. J. Anim. Sci., 63, 1824-1829.

Dikeman M.E., Reddy G.B., Arthaud V.H., Tuma H.J., Koch R.M., Mandigo R.W., Axe J.B., 1986. Longissimus muscle quality, palatability and connective tissue histological characteristics of bulls and steers fed different energy levels and slaughtered at four ages. J. Anim. Sci., 63, 92101.

Dinius D.A., Cross H.R., 1978. Feedlot performance, carcass characteristics and meat palatability of steers fed concentrate for short periods. J. Anim. Sci., 47, 1109-1113.

Dransfield E., Nute G.R., Francombe M.A., 1984. Comparison of eating quality of bull and steer beef. Anim. Prod., 39, 37-50.

Dransfield E., Martin J.F., Bauchart D. Abouelkaram S., Lepetit J., Culioli J., Jurie C., Picard B., 2003. Meat quality and composition of three muscles from French cull cows and young bulls. Anim. Sci., 76, 387-399.

Dumont R., Teissier J.H., Bonnemaire J., Roux M., 1987. Early calving heifers versus maiden heifers for beef production from dairy herds. II. Physicochemical and sensorial characteristics of meat. Liv. Prod. Sci., 16, 21-35.

Dumont R., Roux M., Agabriel J., Touraille C., Bonnemaire J., Malterre C., Robelin J., 1991. Engraissement des vaches de réforme de race Charolaise. Facteurs de variation des performances zootechniques, de la composition tissulaire des carcasses et de la qualité organoleptique de la viande. INRA Prod. Anim., 4, 271-286.

Field R., McCormick R., Balasubramanian V. Sanson D., Wise J., Hixon D., Riley M., Russell W., 1996. Growth, carcass, and tenderness characteristics of virgin, spayed, and single-calf heifers. J. Anim. Sci., 74, 2178-2186.

Fiems L.O., De Campeneere D., De Smet S., Van de Voorde G., Vanacker J.M., Boucque C.V., 2000. Relationship between fat depots in carcasses of beef bulls and effect on meat colour and tenderness. Meat Sci., 56, 41-47.

Fiems L.O., De Campeneere S., Van Caelenbergh W., De Boever J.L., Vanacker J.M.,
2003. Carcass and meat quality in double-muscled Belgian Blue bulls and cows. Meat Sci., 63, 345-352.

Fishell V.K., Aberle E.D., Judge M.D., Perry T.W., 1985. Palatability and muscle properties of beef as influenced by pre slaughter growth rate. J. Anim. Sci., 61, 151-157.

French P., O'Riordan E.G., Monahan F.J., Caffrey P.J., Vidal M., Mooney M.T., Troy D.J. Moloney A.P., 2000. Meat quality of steers finished on autumn grass, grass silage or concentrate based diets. Meat Sci., 56, 173-180.

French P., O'Riordan E.G., Monahan F.J., Caffrey P.J., Mooney M.T., Troy D.J., Moloney A.P., 2001. The eating quality of meat of steers fed grass and/or concentrates. Meat Sci., 57, 379-386.

Frickh J.J., Steinwidder A., Baumung R., 2003. Einfluss von rationgestaltung, geschlecht und mastendmasse auf die fleischqualität von Fleckvieh-Tieren. Züchtungskunde, 75, 16-30.

Garcia-de Siles J.L., Wilson L.L., Ziegler J.H., Watkins J.L., 1982. The effects of slaughter age on growth and carcass traits in an intensively managed crossbred beef herd. Liv. Prod. Sci., 9 , 375-388.

Geay Y., Picard B., Jailler R ., Jailler R., Listrat A., Jurie C., Bayle M.C., Touraille C., 1997. Effets de la nature de la ration sur les performances, les caractéristiques musculaires et la qualité de la viande de taurillons salers. Renc. Rech. Rum., 4, 307-310.

Geay Y., Bauchart D., Hocquette J.F., Culioli J., 2002. Valeur diététique et qualités sensorielles des viandes de ruminants. Incidence de l'alimentation des animaux. INRA Prod. Anim., 15, $37-$ 52.

Gregory K.E., Cundiff L.V., Koch R.M., Dikeman M.E., Koohmaraie M., 1994. Breed effects, retained heterosis, and estimates of genetic and phenotypic parameters for carcass and meat traits of beef cattle. J. Anim. Sci., 72, 1174-1183.

Harrison A.R., Smith M.E., Allen D.M., Hunt M.C., Kastner C.L., Kropf D.H., 1978. Nutritional regime effects on quality and yield characteristics of beef. J. Anim. Sci., 47, 383-388.

Harper G.S., 1999. Trends in skeletal muscle biology and the understanding of toughness in beef. Aust. J. Agric. Res., 50, 1105-1129.

Haurez P., Joulie A., 1994. Influence de la vitesse de croissance et de l'âge à l'abattage des jeunes bovins charolais sur la qualité de la viande. Renc. Rech. Rum., 1, 201-204.

Hennessy D.H., Morris S.G., 2003. Effect of a pre-weaning growth restriction on the subsequent growth and meat quality of yearling steers and heifers. Aust. J. Exp. Agric., 43, 335-341.

Henrickson R.L., Moore R.E., 1965. Effects of animal age on the palatability of beef. Techn. Bull., Oklahoma State University, USA, T-115, $16 \mathrm{p}$.

Hiner R.L., Hankins O.G., 1950. The tenderness of beef in relation to different muscles and age in the animal. J. Anim. Sci., 9, 347-353.

Hoch T., Begon C., Cassar-Malek I., Picard B. Savary-Auzeloux I., 2003. Mécanismes et conséquences de la croissance compensatrice chez les ruminants. INRA Prod. Anim., 16, 49-59. 
Hocquette J.F., Cassar-Malek I., Listrat A., Jurie C., Jailler R., Picard B., 2005. Evolution des recherches sur le muscle des bovins et la qualité sensorielle de leur viande. II. Influence des facteurs d'élevage sur les caractéristiques musculaires. Cah. Agric., 14, 365-372.

Hornick J.L., Van Eenaeme C., Clinquart A., Diez M., Istasse L., 1998. Different periods of feed restriction before compensatory growth in Belgian Blue bulls: I. Animal performance, nitrogen balance, meat characteristics, and fat composition. J. Anim. Sci., 76, 249-259.

Jeremiah L.E., Dugan M.E.R., Aalhus J.L., Gibson L.L., 2003. Assessment of the relationship between chemical components and palatability of major beef muscles and muscle groups. Meat Sci., 65, 1013-1019.

Juniper D.T., Browne E.M., Fisher A.V., Bruant M.J., Nute G.R., Beever D.E., 2005. Intake, growth and meat quality of steers given diets based on varying proportions of maize silage and grass silage. Anim. Sci., 81, 159-170.

Kogel V.J., Augustini C., Pickl M., Edelmann P., 1998. Einfluss von grassilage, heu und maissilage auf dir fleischqualitat von mastfarsen. Zuchtungskunde, 70, 188-195.

Koohmaraie M., Kent M., Shckelford S., Veiseth E., Wheeler T., 2002. Meat tenderness and muscle growth: is there a relationship? Meat Sci., 62 (S), 345-352.

Kopp J., Bonnet M., 1982. Le tissu conjonctif musculaire. Bull. Techn. C.R.Z.V. Theix, INRA, 48, 34-37.

Laborde F.L., Mandell I.B., Tosh J.J., Buchanan-Smith J.G., Wilton J.W., 2002. Effect of management strategy on growth performance, carcass characteristics, fatty acid composition, and palatability attributes in crossbred steers. Can. J. Anim. Sci., 82, 49-57.

Lahucky R., Palanska O., Mojto J., Zaujec K., Huba J., 1998. Effect of pre-slaughter handling on muscle glycogen level and selected meat quality traits in beef. Meat Sci., 50, 389-393.

Lepetit J., 1988. Influence de l'anisotropie et de l'état d'étirement du muscle sur l'analyse des fibres musculaires et conjonctives par une méthode de compression sinusoïdale. IAA, 526-529.

Lepetit J., Culioli J., 1994. Mechanical properties of meat. Meat Sci., 36, 203-237.

Liboriussen T., Bech Andersen B., Buchter L., Kousgaard K., Juel Moller A., 1977. Crossbreeding experiment with beef and dualpurpose sire breeds on Danish dairy cows. IV. Physical, chemical and palatability characteristics of longissimus dorsi and semitendinosus muscles from crossbred young bulls. Liv. Prod. Sci., 4, 31-43.

Maher S.C., Mullen A.M., Moloney A.P., Drennan M.J., Buckley D.J., Jerry J.P., 2004. Colour, composition and eating quality of beef from the progeny of two Charolais sires. Meat Sci., 67, 73-80.

Maltin C., Balcerzak D., Tilley R., Delday M., 2003. Determinants of meat quality: tenderness. Proc. Nut. Soc., 62, 337-347.

Mandell I.B., Buchanan-Smith J.G., Campbell C.P., 1998. Effects of forage $v s$ grain feeding on carcass characteristics, fatty acid composition, and beef quality in Limousin-cross steers when time on feed is controlled. J. Anim. Sci., 76, 2619-2630

Marino R., Albenzio M., Girolami A., Muscio A., Sevi A., Braghieri A., 2006. Effect of forage to concentrate ratio on growth performance, and on carcass and meat quality of Podolian young bulls. Meat Sci., 72, 415-424.

Micol D., Berge P., Dozias D., Lepetit J., Lienard G., Picard B., Renerre M., Robelin J., Touraille C., Geay Y., 1992. Effect of pregnancy and calving on muscle characteristics in cattle. $38^{\text {th }}$ Int. Cong. Meat Sci. Technol., ClermontFerrand, France, 93-96.

Moloney A.P., Fallon R.J., Mooney M.T., Troy D.J., 2004. The quality of meat and fatness of bulls offered ad libitum concentrates, indoors or at pasture. Liv. Prod. Sci., 87, 271-276.

Muir P.D., Smith N.B., Dobbie P.M., Smith D.R., Bown M.D., 2001. Effects of growth pathway on beef quality in 18-month-old Angus and South Devon X Angus pasture-fed steers. Anim. Sci., 72, 297-308.

Muller L., Perobelli Z., Feijo G.L.D., Grassi C., 1992. Cull cow physiological maturity and its effect on carcass and meat quality. $38^{\text {th }}$ Int. Cong. Meat Sci. Technol., Clermont-Ferrand, France, 101-104.

Ockerman H. W., Jaworek D., Van Stavern B. Parrett N., Pierson C. J., 1984. Castration and sire effects on carcass traits, meat palatability and muscle fiber characteristics in Angus cattle. J. Anim. Sci., 59, 981-990.

Office de l'élevage, 2006. La consommation des produits carnés. Septembre 2006, 72p + annexes. (http://www.ofival.fr/consommation /conso-06/conso05.htm)

Ouali A., 1991. Conséquences des traitements technologiques sur la qualité de la viande. INRA Prod. Anim., 4, 195-208.

Oury M.P., 2006. Eléments de différenciation de la qualité sensorielle des viandes liés aux pratiques d'élevage chez la génisse Charolaise. Thèse de doctorat, Université de Bourgogne, ENESAD, 139p.

Ozawa S., Mitsuhashi T. Mitsumoti M. Matsumoto S., Itoh N., Itagaki N., Kohno Y., Dohgo T., 2000. The characteristics of muscle fiber types of longissimus thoracis muscle and their influences on the quantity and quality of meat from Japanes Blacks steers. Meat Sci., 54, 65-70.

Patterson D.C., Moore C.A., Moss B.W., Kilpatrick D.J., 2002. Parity-associated changes in slaughter weight and carcass characteristics of $3 / 4$ Charolais crossbred cows kept on a lowland grass/grass silage feeding and management system. Anim. Sci., 75, 221-235.

Picard B., Robelin J., Geay Y., 1995. Influence of castration and postnatal energy restriction on the contractile and metabolic characteristics of bovine muscle. Ann. Zootech., 44, 347-357.

Perry D., Thompson J.M., 2005. The effect of growth rate during back grounding and finishing on meat quality traits in beef cattle. Meat Sci., $69,691-702$

Purchas R.W., Burnham D.L., Morris S.T., 2002. Effects of growth potential and growth path on tenderness of beef longissimus muscle from bulls and steers. J. Anim. Sci., 80, 3211-3221.

Purslow P.P., 1994. The structural basis of meat toughness : what role does the collagenous component play? Proc. Int. Cong. Meat Sci. Technol., 40, 27-34.

Reagan J.O., Stribling K.V., Carpenter J.A., Campion D.R., 1981. Microbiological, vacuum packaging and palatability attributes of beef produced at varied levels of forages and grain. J. Anim. Sci., 53, 1482-1488.
Realini C.E., Duckett S.K., Brito G.W. , Dalla Rizza M., De Mattos D., 2004. Effect of pasture $v s$ concentrate feeding with or without antioxidants on carcass characteristics, fatty acid composition, and quality of Uruguayan beef. Meat Sci., 66, 567-577.

Renand G., 1988. Variabilité génétique de la croissance musculaire et conséquences sur les qualités de la viande chez les bovins. INRA Prod. Anim., 1, 115-121.

Renand G., Touraille C., Geay Y., Berge P., Lepetit J., Picard B., 1997. Variabilité des qualités organoleptiques de la viande bovine en relation avec les caractéristiques musculaires. Renc. Rech. Rum., 7, 311-314.

Renand G., Picard B., Touraille C., Berge P., Lepetit J., 2001. Relationships between muscle characteristics and meat quality traits of young Charolais bulls. Meat Sci., 59, 49-60.

Rhee M.S., Wheeler T.L., Shackelford S.D., Koohmaraie M., 2004. Variation in palatability and biochemical traits within and among eleven beef muscles. J. Anim. Sci., 82, 534-550.

Roux M., Dumont R., Agabriel J., Bonnemaire J., Micol D., 1993. Engraissement des vaches de réforme de race Charolaise. Effet d'une suralimentation protéique sur les performances d'engraissement et les caractéristiques physico-chimiques musculaires. INRA Prod. Anim., 6, 237-248.

Ryan W.J., 1990. Compensatory growth in cattle and sheep. Nut. Abst. Rev., Ser. B, 60, $653-$ 664

Salé P., 1971. Evolution de quelques propriétés mécaniques du muscle pendant la maturation. Bull. Tech. C.R.Z.V. Theix, INRA, 6, 35-44

Sami A.S., Augustini C., Schwars F.J., 2004. Effects of feeding intensity and time on feed on performance, carcass characteristics and meat quality of Simmental bulls. Meat Sci., 67, 195201.

Schnell T.D., Belk K.E., Tatum J.D., Miller R.K., Smith G.C., 1997. Performance, carcass, and palatability traits for cull cows fed high energy concentrate diets for $0,14,28,42$, or 56 days. J. Anim. Sci., 75, 1195-1202.

Schwarz F.J., 2003. Zum einfluss der fütterung auf dir rindfleishqualität. Züchtungskunde, $75,357-367$.

Shackelford S.D., Koohmaraie M., Cundiff L.V., Gregory K.E., Rohrer G.A., Savell J.W., 1994. Heritabilities and phenotypic and genetic correlations for bovine postrigor calpastatin activity, intramuscular fat content, WarnerBratzler shear force, retail product yield and growth rate. J. Anim. Sci., 72, 857-863.

Shackelford S.D., Koohmaraie M., Wheeler T.L., 1995. Effects of slaughter age on meat tenderness and USDA carcass maturity scores of beef females. J. Anim. Sci., 73, 3304-3309.

Shemeis A.R., Liboriussen T., Bech Andersen B., Abdallah O. Y., 1994. Changes in carcass and meat quality traits of Danish Friesian cull cows with the increase of their age and body condition. Meat Sci., 37, 161-167.

Sinclair K.D., Cuthbertson A., Rutter A., Franklin M.F., 1998. The effects of age at slaughter, genotype and finishing system on the organoleptic properties and texture of bull beef from suckled calves. Anim. Sci., 66, 329-340.

Sinclair K.D., Lobley G.E., Horgan G.W., Kyle D.J., Porter A.D., Matthews K.R., Warkup C.C., Maltin C.A., 2001. Factors influencing beef 
eating quality. 1. Effects of nutritional regimen and genotype on organoleptic properties and instrumental texture. Anim. Sci., 72, 269-277.

Tarrant P.V., 1998. Some recent advances and future priorities for the meat industry. Meat Sci., 49, Suppl. 1, S1-S6.

Tatum J.D., Smith C.C., Berry B.W., Murphey C.E., Williams F.L., Carpenter Z.L., 1980. Carcass characteristics, time on feed and cooked beef palatability attributes. J. Anim. Sci., 50, 833-840.

Tatum J.D. 1981. Is tenderness nutritionally controlled? Proc. Reciprocal Meat Conf., 34, 65.

Touraille C., 1981. La dégustation de la viande et des produits carnés. Viandes et Produits Carnés, 2, 29-34.

Touraille C., 1982. Qualité des viandes de taurillons : évolution avec l'âge des caractéristiques physico-chimiques des muscles - la tendreté de la viande. Bull. Tech. C.R.Z.V. Theix, INRA, 48, 37-41.
Van Koevering M.T., Gill D.R., Owens F.N., Dolezal H.G., Strasia C.A., 1995. Effect of time on feed on performance of feedlot steers, carcass characteristics, and tenderness and composition of longissimus muscles. J. Anim. Sci., 73, 21-28.

Van Vooren T., Van Hoof J., Vereecke D., Istasse L., Dufrasne I., Deswysen A., 1992. Influence of nutrition on meat quality in double muscle bulls. $38^{\text {th }}$ Int. Cong. Meat Sci. Technol., Clermont-Ferrand, France,153-156.

Vestergaard M., Therkildsen M., Henckel P., Jensen L.R., Andersen H.R., Sejrsen K., 2000. Influence of feeding intensity, grazing and finishing feeding on meat and eating quality of young bulls and the relationship between muscle fibre characteristics, fibre fragmentation and meat tenderness. Meat Sci., 54, 187-195.

Waggoner A.W., Dikeman M.E., Brethour J.R., Kemp K.E., 1990. Performance, carcass, cartilage calcium, sensory and collagen traits of longissimus muscles of open versus 30 -monthold heifers that produced one calf. J. Anim. Sci., 68, 2380-2386.
Warner K. F., 1928. A study of the factors which influence the quality and the palatability of meat. U.S. Dept. Agric. Nat. Coop. Pro., Coop. Bur Animal Ind. Rev. Ed.

Wertz E., Berger L.L., Walker P.M., Faulkner D.B., McKeith F.K., Rodriguez-Zas S., 2001. Early weaning and post weaning nutritional management affect feedlot performance of Angus $x$ Simmental heifers and the relationship of 12 th rib fat and marbling score to feed efficiency. J. Anim. Sci., 79, 1660-1669.

Wu J.J., Kastner C.L., Hunt M.C., Kropf D.H., Allen D.M., 1981. Nutritional effects on beef collagen characteristics and palatability. J. Anim. Sci., 53, 1256-1261.

Xie Y.R., Busboom J.R., Cornforth D.P., Shenton H.T., Gaskins C.T., Johnson K.A., Reeves J.J., Wright R.W., Cronrath J.D., 1996. Effects of time on feed and post-mortem ageing on palatability and lipid composition of crossbred Wagyu beef. Meat Sci., 43, 157-166.

\section{Résumé}

La tendreté est un paramètre important dans l'évaluation de la qualité de la viande bovine. Elle est sous la dépendance d'une combinaison complexe de paramètres, parmi lesquelles les facteurs technologiques jouent un rôle essentiel. Cependant, lorsque l'environnement péri-abattage est maîtrisé, il demeure une variabilité de la tendreté des viandes, mal perçue par le consommateur, et qui peut être attribuée aux facteurs d'élevage. Au sein d'une même race, le type morphologique n'a pas d'impact sur la tendreté des viandes et la force de cisaillement des muscles. La tendreté et la force de cisaillement de la viande de bœuf et de taurillon n'évoluent pas avant l'âge de 2 ans. Avant 35 mois, elles n'évoluent que très rarement chez la femelle mais au-delà, un effet négatif de l'âge peut parfois être mis en évidence. Parmi les facteurs liés au mode de conduite, il apparaît que la gestation et le vêlage ne détériorent pas la tendreté de la viande et que l'activité physique est favorable à la tendreté des muscles locomoteurs sans influencer celle des muscles non locomoteurs. Concernant la conduite alimentaire, il faut retenir que la croissance compensatrice, l'augmentation du niveau alimentaire et de la durée de finition peuvent influencer favorablement la tendreté de la viande, mais ces effets positifs restent à confirmer. La nature de l'alimentation a également des effets limités sur la tendreté de la viande, variables selon les aliments étudiés et les conditions d'expérimentation.

\section{Abstract}

\section{Effect of rearing management practices on tenderness of bovine meat}

Tenderness is an important criterion when evaluating bovine meat quality. This descriptor depends on a complex combination of parameters, in which technological factors are essential. Nevertheless, when post-slaughter conditions are fixed, wide variations among meat tenderness still exist. This variability may be linked to rearing factors.

When considering animals of a determined breed, the morphological type has no impact on meat tenderness and muscle shear force. Tenderness and shear force of steers and bulls are not modified by age at slaughter before 24 months. Before 35 months, age at slaughter has no impact on heifer meat tenderness. Beyond, an increase of slaughter age may have an unfavourable impact on meat tenderness of cows. Among rearing factors, pregnancy and calving do not damage meat tenderness. Exercise has a positive impact on the tenderness of locomotive muscles but no influence on the tenderness of non-locomotive muscles. Considering the feeding factors, it appears that increasing the feeding level or lengthening the finishing period may have a positive impact on meat tenderness in a few studies. Compensatory growth may also have in some studies a positive influence, which needs to be confirmed. The influence of feeding on meat tenderness is also limited and variable depending on the feeding type and the experimental conditions.

OURY M.-P., PICARD B., ISTASSE L., MICOL D., DUMONT R., 2007. Mode de conduite en élevage et tendreté de la viande bovine. INRA Prod. Anim., 20, 309-326. 
